\title{
Modelling of steady-state fluid flow in 3D fractured isotropic porous media: Application to effective permeability calculation
}

\author{
Minh-Ngoc Vu ${ }^{1,2, * \dagger}$, Ahmad Pouya ${ }^{1,}$, Darius M. Seyedi ${ }^{2}$ \\ ${ }^{1}$ Université Paris-Est, Laboratoire Navier (ENPC-IFSTTAR-CNRS) 77455 Marne la Vallée, France \\ ${ }^{2}$ BRGM, Risks Division, F-45060 Orléans Cedex 2 France \\ ${ }^{*, \dagger}$ Corresponding author: vum@ cermes.enpc.fr \\ Tel.: +331641535 35; fax.: +33164153562
}

\begin{abstract}
SUMMARY
In this paper, 3D steady-state fluid flow in a porous medium with many intersecting fractures is derived numerically by using collocation method. Fluid flow, in the matrix and fractures, are described by Darcy's law and Poisseuille's law, respectively. The recent theoretical development presented a general potential solution to model the steady-state flow in fractured porous media under a far-field condition. This solution is a hypersingular integral equation with pressure field in the fracture surfaces as the main unknown. The numerical procedure can resolve the problem for any form of fractures and also takes into account the interactions and the intersection between fractures. Once the pressure field, and then the flux field in fractures have been determined, the pressure field in the porous matrix is computed completely. The basic problem of a single fracture is investigated and a semi-analytical solution is presented. Using the solution obtained for a single fracture, Mori-Tanaka and self-consistent schemes are employed for upscalling the effective permeability of 3D fractured porous media.
\end{abstract}

KEYWORDS: Fractured porous media; steady-state fluid flow; effective permeability; Upscaling; Integral Equation; Poiseuille's flow.

\section{INTRODUCTION}

In the last two decades, there has been an intensive investigation in the fluid flow analysis in cracked porous media or fractured rock masses, due to the modelling requirements for important industrial applications such as: underground radioactive waste repositories, natural oil/gas recovery, geological $\mathrm{CO}_{2}$ storage, geothermal energy, etc. However, determining effective permeability for fractured rock formations is one of the most challenging problems that are faced by geomechanical researchers and geotechnical engineers. Effective continuum is a simple and efficient approach to study the macroscopic properties of fractured and heterogeneous media by taking over a representative elementary volume. Discrete Fracture Network (DFN) model has been a powerful approach to simulate the flow in the natural fractured reservoirs for a long time [1-4]. DFN is introduced in several commercial softwares as UDEC (Itasca) [5], Fraca [6], etc. Nevertheless, the matrix is supposed to have negligible porosity and permeability in DFN models. A common approach to overcome this limit is dual porosity/dual permeability model that was first proposed by Barenblatt et al. [7]. In this framework, fracture and porous matrix are considered as two interactive continua. This 
approach has been widely used for modelling fluid flow in fractured materials. A brief review of several flow models can be consulted in Jafari and Babadagli [4]. Different numerical techniques, such as finite element method (FEM) [8-11] and finite volume method (FVM) [12-16] have been used to resolve fluid flow equations in fractured porous media.

Modelling of the steady-state flow in a fractured porous media under a far-field condition constitutes a key problem for effective permeability determination. In this work, we investigate numerically the flow around several fractures embedded in an infinite body described by a uniform gradient pressure at infinity. The fracture is modelled by a zero thickness surface with no pressure jump between two faces; i.e., having an infinite transverse conductivity. In order to upscale effective permeability, it is interesting to consider the solution of flow around an isolated fracture. This problem can be derived by using the Darcy's ellipsoidal inclusion model [17-19]. Nonetheless, Poiseuille's law is a more common model to describe the flow in a single fracture in the physical rock area. A comparison between these two models can be seen in Pouya and $\mathrm{Vu}[20]$.

Potential solution based on singular integral equation is proposed recently to study the steady-state flow in an infinite fractured porous medium with homogeneous permeability matrix. The 2D problem is studied by Liolios and Exadaktylos [21]; Pouya and Ghabezloo [22]; Pouya and $\mathrm{Vu}$ [23]. In these studies, the effective permeability is computed analytically by Pouya and Ghabezloo [22] and numerically by Pouya and $\mathrm{Vu}$ [23]. The potential solution was extended to 3D problem by Pouya [24], which reveals the pressure field in porous matrix as a function of the infiltration through the curved fracture surfaces. Therefore, this advance of mathematical formulation allows us to decrease dimension of the discretised domain: 3D (pressure field in the whole body) to 2D (infiltration through the fracture surfaces). Pouya and $\mathrm{Vu}$ [20] used this potential solution to establish a semi-analytical solution for a superconductive elliptical discshaped fracture governed by Poiseuille's law flow. Moreover, a closed-form solution for a superconductive circular disc fracture is derived in this work [20]. The obtained solution is then used to estimate the effective permeability. However, any analytical solution for the general case of the fracture with a finite conductivity does not exit. Hence, numerical resolution is naturally imposed.

This paper focuses on singular integral equation method to resolve the potential equation in 3D fractured porous media. Some examples with several intersecting fractures are presented. Numerical solution for a single fracture obtained in this way permits us to establish a semi-analytical description of infiltration through the fracture as a function of fracture conductivity, matrix permeability and fracture geometry. Then, Mori-Tanaka and self-consistent schemes are employed to estimate the effective permeability of a fractured porous media by using this semi-analytical solution. The superconductive fracture is always used in this work as a reference problem to make reader confident on the results.

\section{Notations}

In the following, light-face (Greek or Latin) letters denote scalars; underlined letters denote vectors, bold-face letters designate second rank tensors or double-index matrices. The scalar 
product of two vectors $\underline{a}$ and $\underline{b}$ is labelled as $\underline{a} . \underline{b}$. For second rank tensors, the tensor transposed from $\boldsymbol{A}$ is denoted $\boldsymbol{A}^{\mathrm{T}}$, the matrix product is labelled as $\boldsymbol{A} \boldsymbol{B}$ and the determinant as $|\boldsymbol{A}|$. The operation of $\boldsymbol{A}$ on $\underline{a}$ is labelled as $\boldsymbol{A}$. $\underline{a}$. The convention of summation on repeated indices is not used for Latin indices $(i, j, k \ldots)$ that number surfaces, lines, etc., and are noted indifferently as subscript or superscript. $\nabla$ represents the gradient and $\Delta$ the Laplace operator for a scalar field and $(\nabla$.$) the divergence for a vector field.$

\section{GOVERNING EQUATIONS}

Considering a reference volume $\Omega$ of an infinite three-dimensional porous media having isotropic permeability matrix $\boldsymbol{k}=k \boldsymbol{\delta}$ and containing a family of fractures numbered by $j$ and noted $\Gamma^{j}$ (Fig. 1). Each fracture surface $\Gamma^{j}$ is defined by a smooth function $\underline{z}^{j}$ of the curvilinear parameters $\left\{s_{\alpha}\right\}(\alpha=1,2)$. Two or more fractures can intersect along an intersection line and three or more fractures can cross at an intersection point. The intersection lines and the border of fracture surfaces $\partial \Gamma$ constitute a set of singular points denoted by $S$ [24]. The set of points on the fracture surface is denoted by $\Gamma$. Therefore $\mathrm{S} \subset \Gamma$ and rock matrix corresponds to $\Omega-\Gamma$. A far-field linear pressure defined as $p_{\infty}(\underline{x})=\underline{A} . \underline{x}$ is applied to the domain $\Omega$, with $\underline{A}$ a constant pressure gradient.

The fluid flow in the matrix is governed by Darcy's law:

$\forall \underline{x} \in \Omega-\Gamma \quad \underline{v}(\underline{x})=-k(\underline{x}) \cdot \nabla p(\underline{x})$

where, $\underline{v}(\underline{x})$ and $p(\underline{x})$ are local fluid velocity and pressure, respectively.

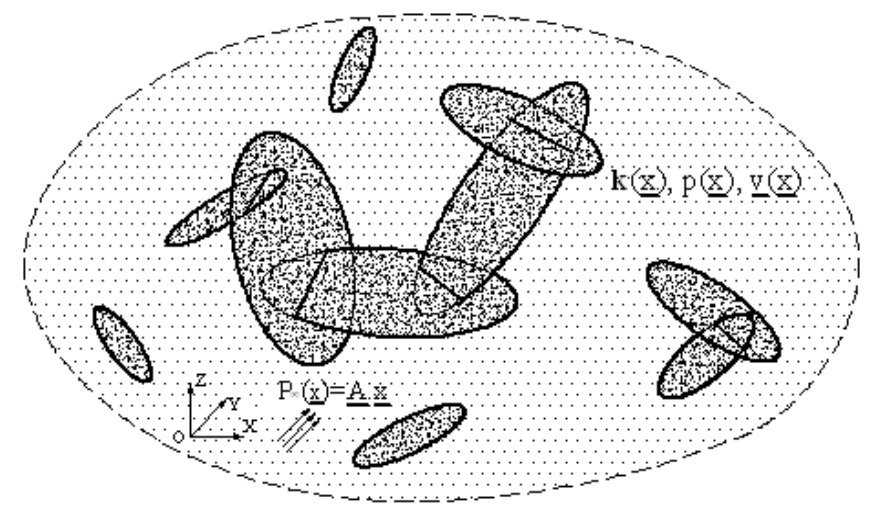

Fig. 1 Porous medium containing intersecting fractures

In absence of point sources, continuity equation of fluid in the matrix reads:

$\forall \underline{x} \in \Omega-\Gamma \quad \nabla \cdot \underline{v}(\underline{x})=0$

As mentioned by Guéguen and Palciauskas [25], Poiseuille's law is the most common model for flow in a single fracture. This law establishes a linear relation between the flux vector and the in-plane pressure gradient in the fracture as follows:

$\forall s \in \Gamma \quad \underline{q}(\underline{s})=-\boldsymbol{c}(\underline{s}) \cdot \nabla_{s} p(\underline{s})$

where, $\boldsymbol{c}$ is the hydraulic conductivity and consists of a tensor of $2 \times 2$ dimension in the plane of fracture and is assumed to be isotropic (i.e., $\boldsymbol{c}=c \boldsymbol{\delta}$ ). By neglecting roughness effect, the hydraulic conductivity of a fracture $c$ is deduced by the classical cubic law. 
Hence $c$ depends on the fracture aperture $e$ and the fluid dynamic viscosity $\mu$, as $c=e^{3} /(12 \mu)$. However, real rock fractures have rough walls, variable aperture and asperity areas. To simplify the problem, a single average value, called hydraulic aperture $e^{*}$, is usually employed to characterize the mechanical aperture $e$ of an individual fracture. The empirical relations between these two variables can be found in a great amount of works in the literature. A review of these models is available in Zhou et al. [26].

The in-plane pressure gradient is defined by the flowing formulation:

$$
\nabla_{s} p(s)=\frac{\partial p}{\partial s_{1}} \underline{t}_{1}+\frac{\partial p}{\partial s_{2}} \underline{t}_{2}
$$

and, $\left(\underline{t}_{1}, \underline{t}_{2}\right)$ defined by $\underline{t}_{\alpha}=\partial \underline{z} / \partial s_{\alpha}$ are two perpendicular unit vectors tangent to $\Gamma$ (Fig. 2 )

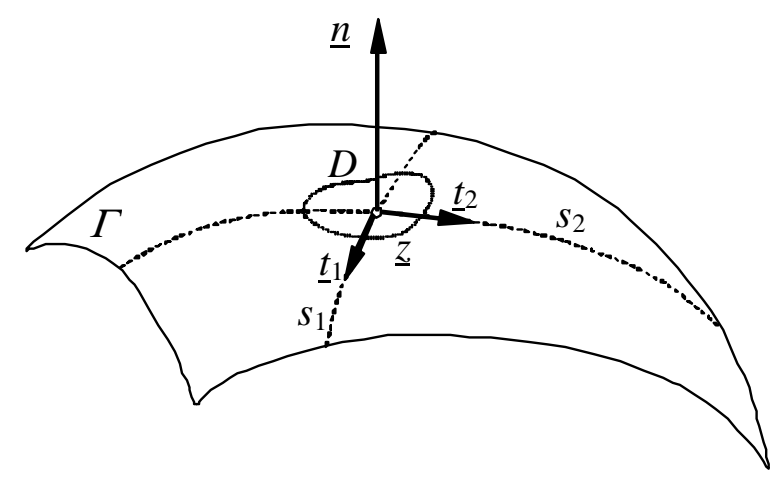

Fig. 2 Curvilinear parameters [24]

The fracture-matrix mass exchange at a regular point on the fracture $\Gamma-S$ (excluding the intersection points) is widely described in the literature $[7,27,28,13,16]$. This law generally has been obtained by considering mass balance in a small volume $D$ around a point $\underline{z}$. Using the scheme presented in Fig. 3a, the mass conservation reads [24]:

$$
\llbracket \underline{v}(\underline{z}) \rrbracket \cdot \underline{n}(\underline{s})+\nabla_{s} \cdot \underline{q}(\underline{s})=0
$$

where, $\underline{n}$ is the normal unit vector to the fracture surface, pointing from $\Gamma^{-}$to $\Gamma^{+} \cdot \underline{v}^{-}$and $\underline{v}^{+}$are the fluid velocity in the matrix at the point $\underline{z}$ of lower and upper fracture surface, respectively. $\llbracket \underline{v}(\underline{z}) \rrbracket=\underline{v}^{+}(\underline{z})-\underline{v}^{-}(\underline{z})$ designates the discontinuity or jump of fluid velocity across the fracture. $\nabla_{s} \cdot q(\underline{s})$ represents the surface divergence. If the curvature effect of surface is neglected [24], the surface divergence reads:

$$
\underline{\nabla}_{s} \underline{q}(s)=\frac{\partial q_{1}}{\partial s_{1}}+\frac{\partial q_{2}}{\partial s_{2}}
$$

Mass balance at intersection lines between several fractures surfaces is an important issue in studying the fluid flow in the fractured porous media. Pouya [24] presented a rigorous way to obtain the closed-form formulation of mass balance at a singular point. This relation is similar to one that can be found for a fracture network in an impermeable medium. 


$$
\sum_{j} \underline{q}_{j} \cdot \underline{m}_{j}=0
$$

where, $q_{j}$ is the infiltration vector on $\Gamma_{j}$ and $\underline{m}_{j}$ tangent normal vector of the intersection line on $\Gamma_{j}$ pointing outward from the domain as seen in Fig. $3 b$.
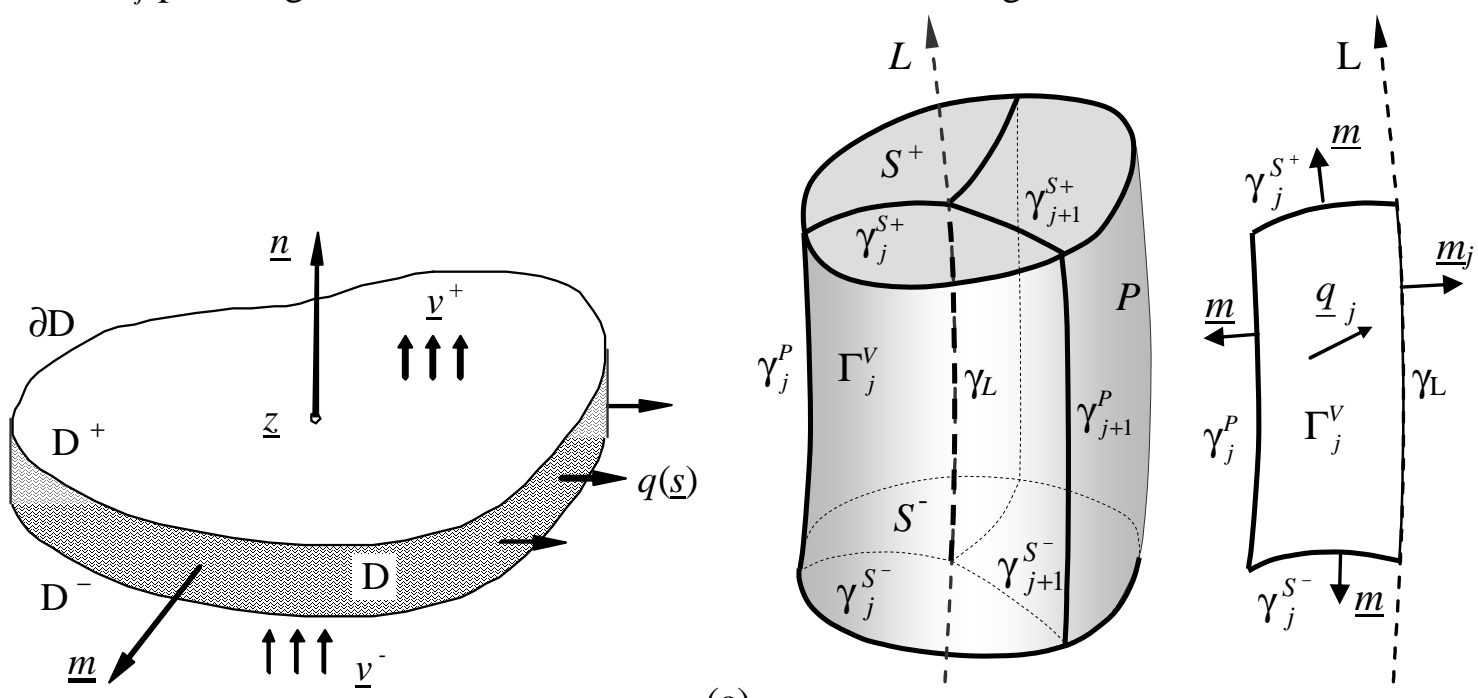

(a)

(b)

Fig. 3 Masse exchange between matrix and fracture [24]: (a) at a regular point and (b) on an intersection line

The pressure field $p(\underline{x})$ in a fractured porous domain, under a far-field constant pressure gradient, must satisfy the constitutive equations (1), (3) and mass balances equations (2), (5), (7) and boundary conditions. For fractures with infinite transversal transmissivity (no pressure jump between their two sides), Pouya [24] proposed a general pressure potential solution as a function of the infiltration through the fractures such as:

$$
p(\underline{x})=p_{\infty}(\underline{x})+\frac{1}{4 \pi k} \sum_{j} \int_{\Gamma^{j}} \frac{\underline{q}^{j}(\underline{s}) \cdot\left(\underline{x}-\underline{z}^{j}(s)\right)}{\left\|\underline{x}-\underline{z}^{j}(s)\right\|^{3}} d s
$$

For the effective permeability estimation purposes, the numerical calculation is only applied for plane surface factures in the present work. Nevertheless, equation (8) is valid for the curved intersecting fracture surfaces with arbitrary forms.

Introducing equation (3) into (8), a hypersingular integral equations is obtained, in which the pressure field on fractures is the main unknown. The proposed flow model makes an important advantage by reducing a three-dimensional problem to two-dimensional one. Hence, 2D mesh is only generated on the fracture surfaces in the first stage to obtain the pressure and the infiltration solutions on the fractures, and then, these results are used again to compute the pressure field in the whole domain in the second stage. Unlike FEM and FVM, which pass by volume discretization and require 3D meshes in the whole space.

\section{NUMERICAL RESOLUTION}

FEM is very efficient for solving finite-domain problems, but it is less convenient to 
deal with infinite domain problems. Integral equation method or boundary element method is an efficient alternative that has been extensively used for solving infinite or half-plane problems [29,30]. This section describes a numerical procedure to solve equation (8) based on collocation method. The principle resolution of integral equation by collocation method can be found in Bonnet [31]. A robust methodology is proposed in this work to achieve fast numerical calculations. The numerical procedure is presented for solving a general problem to model the flow in an infinite isotropic medium containing several intersecting curved fracture surfaces under a uniform far-field pressure gradient. Some results of pressure field on the fractures are illustrated for many intersecting fractures or a curved surface at the end of this section to demonstrate the capacity of the developed numerical code.

\subsection{Geometry discretization and variables interpolation}

A mesh is generated on the surface of all fractures. It is constituted by $N$ nodes and $M$ triangular linear elements numbered $m$ and denoted $E_{m}$. This mesh must be consistent at the intersection lines between the fractures. By replacing the infiltration by equation (3), an approximated form for equation (8) can be obtained:

$$
p(\underline{x}) \approx p_{\infty}(\underline{x})-\frac{c}{4 \pi k} \sum_{m=1}^{M} I_{m}(\underline{x})
$$

where, $I_{m}$ is an elementary integral:

$$
I_{m}(\underline{x})=\int_{E_{m}} \frac{\nabla p(\underline{z}) \cdot(\underline{x}-\underline{z}(s))}{\|\underline{x}-\underline{z}(s)\|^{3}} d s
$$

The choice of the interpolation function for the elements at the boundary of fractures is based on the velocity profile in this area. Regarding one-dimensional singular integral equation over a finite interval, many authors [32-34] characterized the solution profile at the extremity points, which varies as $r^{1 / 2}$ in vicinity of these points. Pouya and Ghabezloo [22] derived the problem of fluid flow around an isolated superconductive crack line located at the interval $[-1,1]$ on the $O x$-axis and under a constant gradient of

pressure at infinity. A closed-form solution of flux is found, which varies as $\sqrt{1-r^{2}}$ in the fracture. Pouya and $\mathrm{Vu}[23]$ used the interpolation of discharge $q(s)$ corresponding to a variation according to $s^{1 / 2}$ for the general case of Poiseuille's fracture, with $s$ the distance from extremity point. In two dimensional, Wolfe [35] and Boersma and Danicki [36] considered the problem of the electrostatic potential due to a charged elliptic disk. The solution also varies as $r^{1 / 2}$ closed to disk border, with $r$ the distance from the disk border. Recently, Pouya and $\mathrm{Vu}$ [20] analytically derived the problem presented in this work for a single superconductive circular disc fracture. They showed the infiltration solution varies as $\sqrt{1-r^{2}}$ perpendicular to the fracture border.

As a result, three types of element are distinguished: Type 0,1 and 2 correspond to the element that has no node, one and two nodes on the border of fractures respectively (Fig. 4). Element of type 0 is considered as isoparametric, i.e. a linear interpolation of pressure is used that leads to a constant flux $q$ on that elements. On the other hand, inspiring from closed-form solution of two-dimensional and three-dimensional problems, the interpolation function of flux vector is chosen for element of types 1 and 2, which 
varies as $r^{1 / 2}$ in the perpendicular direction of the facture boundary and be constant in the other direction in each element of those types as depicted in Fig. 5.

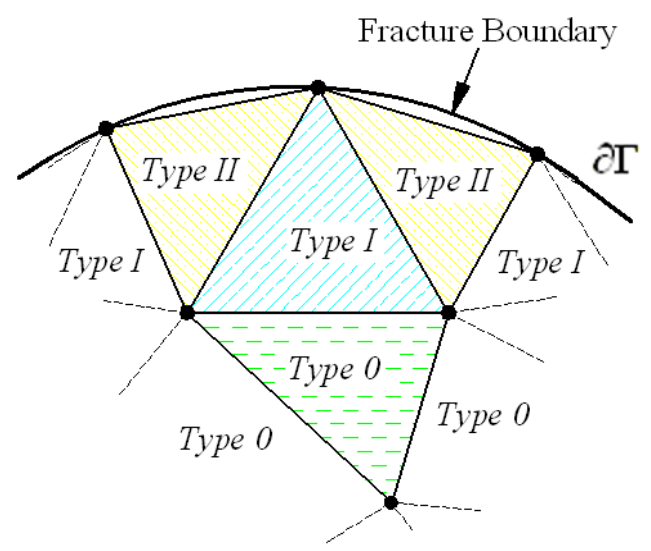

Fig. 4 Three different types of element
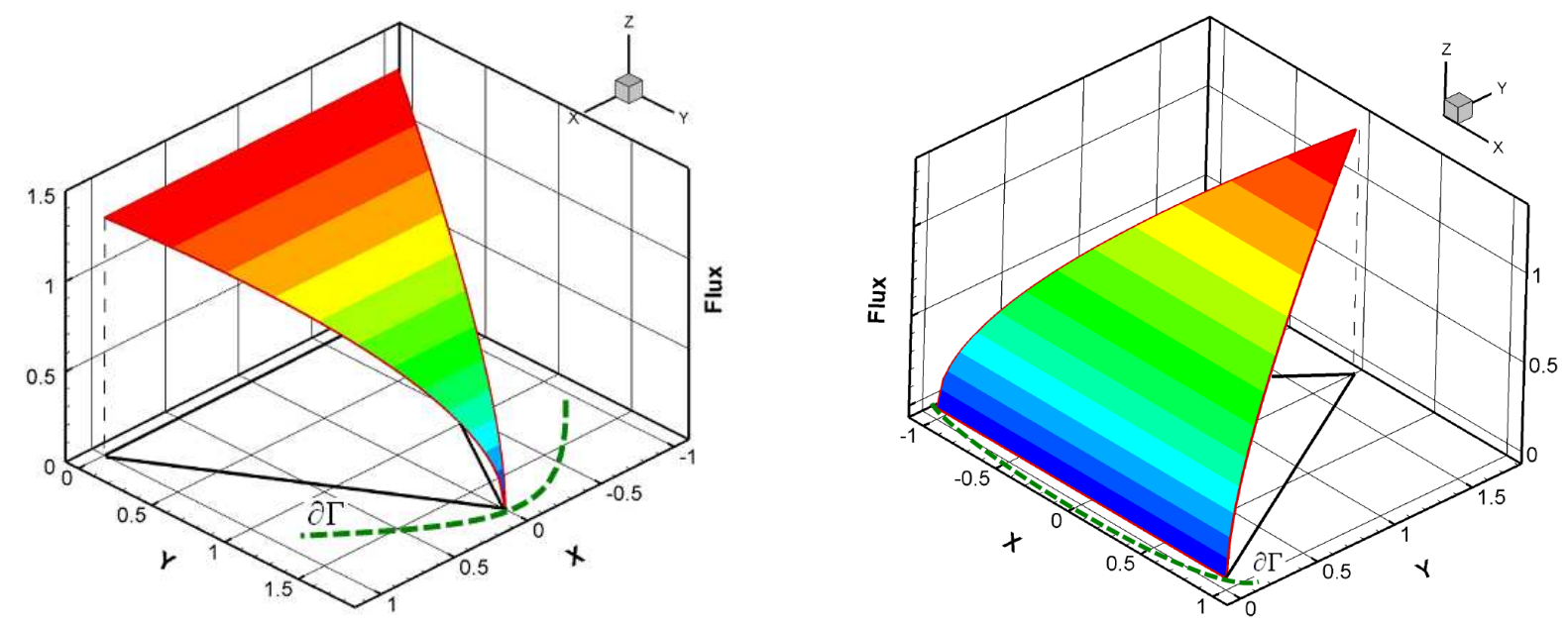

Fig. 5 Interpolation function of flux flow in the element of type 1 (left) and of type 2 (right)

Interpolation functions of pressure on the elements of type 1 and 2 can be deduced from equation (3) and its formulations in global coordinates systems is presented in Appendix A.

To simplify the notation, $\psi_{k}(\underline{x})$ is used to show the interpolation functions of three types of elements throughout this text.

\subsection{Construction and resolution of linear system of Equations}

The collocation method consists in forcing the integro-differential equation (9) to be verified exactly at collocation points $\underline{x}_{c} . M$ collocation points are chosen as the set of barycenters of $M$ triangular elements and are numbered as the elements. Therefore, the discretization of equations, for collocation point $\underline{x}_{c}^{i}$ on the element $i$, is performed by transferring the variables interpolations:

$$
p\left(\underline{x}_{c}^{i}\right) \approx p_{\infty}\left(\underline{x}_{c}^{i}\right)-\frac{c}{4 \pi k} \sum_{m=1}^{M} I_{m}\left(\underline{x}_{c}^{i}\right)
$$


such as:

$$
\sum_{k=1}^{3} \psi_{k}\left(\underline{x}_{c}^{i}\right) \cdot p_{k}^{i} \approx \underline{A} \cdot \underline{x}_{c}^{i}-\frac{c}{4 \pi k} \sum_{m=1}^{M} \sum_{k=1}^{3} \int_{E_{m}} \frac{\nabla\left(\psi_{k}(\underline{z}) \cdot p_{k}^{m}\right) \cdot\left(\underline{x}_{c}^{i}-\underline{z}(s)\right)}{\left\|\underline{x}_{c}^{i}-\underline{z}(s)\right\|^{3}} d s
$$

This equation is first written for $M$ collocation points, and then, assembly operation based on two nested loops over all collocation points and all elements lead to:

$$
\text { H.P }=\boldsymbol{G}
$$

where, $\boldsymbol{G}(M \times 1)$ is the column of pressure prescribed at infinity $p_{\infty}(\underline{x})$ at $M$ collocation points, $\boldsymbol{P}(N \times 1)$ the column of nodal pressures or unknowns, and $\mathbf{H}(M \times N)$ the matrix of coefficients obtained by elementary integration calculation explained in the next section.

It should be noted that the system of Equations (13) consists of $M$ equations and $N$ unknowns with $M>N$. Indeed, most of meshes built on the surface of a set of fractures contain more elements than nodes. This situation is always seen for fine meshes. However, the approximated solution could be computed by a least squares fitting method minimizing $|\mathbf{H} . \boldsymbol{P}-\boldsymbol{G}|^{2}$.

\subsection{Elementary integral calculation}

Equation (12) contains an elementary integral:

$$
I_{m k}=\int_{E_{m}} \frac{\nabla\left(\psi_{k}(\underline{z}) \cdot p_{k}^{m}\right) \cdot\left(\underline{x}_{c}^{i}-\underline{z}(s)\right)}{\left\|\underline{x}_{c}^{i}-\underline{z}(s)\right\|^{3}} d s \text { and } I_{m}=\sum_{k=1}^{3} I_{m k}
$$

This integral is singular if $\underline{x}_{c}^{i} \in E_{m}$.i.e. $i=m$ and regular if $\underline{x}_{c}^{i} \notin E_{m}$ or $\mathrm{i} \neq \mathrm{m}$. The singular elementary integrals are evaluated in Cauchy Principal Value meaning [37]. In the boundary element method field, computation of regular elementary integral is usually based, as well as in finite element method, on the use of Gaussian quadrature formulas. However, ordinary numerical method would not yield reliable and consistent solutions for evaluating singular elementary integrals since the singularities arise from the kernels of integrals. Several powerful schemes are applied to compute accurately these integrals such as: integration by parts [38], Limit process [39], and transformation method [31, $40,41]$. The numerical difficulty appears when the collocation point is too close to the element, so the integral is not strictly singular but nearly singular. Boundary element method researchers have proposed various numerical schemes or semi-analytical approaches to derive such nearly singular integrals. A brief review of some techniques can be found in Liu et al. [42].

In this work, the elementary integral may be computed by different ways, namely: analytical, numerical and semi-analytical depending on the relative position of collocation point $\underline{x}_{\mathrm{c}}$ regarding the element $E$ and the type of element (i.e. interpolation function). .

\section{Analytical scheme}

Analytical procedure is developed to calculate the singular elementary integral over element of type 0 (Fig. 6a). The treatment technique of singularity is based on the 
introduction of a polar coordinate system centred at $\underline{x}_{c}$ in element $E$ :

$$
\underline{z}=\underline{x}_{c}+\rho \underline{\phi}
$$

where, $\underline{\phi}=(\cos \theta, \sin \theta)$. Linear interpolation of pressure over element leads that the gradient of interpolation functions is constant, thus, we suppose $\underline{\nabla}\left(\psi_{k}(\underline{z})\right)=\underline{\zeta}_{k}=$ const . Element $E$ is divided into four parts: a small circle $C$ of radius $\varepsilon$ centred at $\underline{x}_{c}$ and three sub-triangles $T_{l}(l=1,2,3)$.

Rewriting integral (14) in polar coordinate yields:

$$
I_{m k}=\sum_{k=1}^{3} p_{k}^{m} \underline{\zeta}_{k}\left(\int_{C} \underline{\phi} \frac{d \rho}{\rho} d \theta+\sum_{l=1}^{3} \int_{T_{l}} \underline{\phi} \frac{d \rho}{\rho} d \theta\right)
$$

then,

$$
I_{m k}=\sum_{k=1}^{3} p_{k}^{m} \underline{\zeta}_{k}\left(\int_{0}^{\varepsilon} \frac{d \rho}{\rho} \int_{0}^{2 \pi} \underline{\phi} d \theta+\sum_{l=1}^{3} \int_{\theta_{l 1}}^{\theta_{l 2}} \underline{\phi} d \theta \int_{\varepsilon}^{\rho_{l}(\theta)} \frac{d \rho}{\rho}\right)
$$

where, the parameter of each sub-triangle $T_{l}(l=1,2,3)$ in equation (17) can be found in Fig. 7. As expected, the first integral in this Equation is a singular integral which is vanishing due to $\int_{0}^{2 \pi} \phi d \theta=0$. The second integral is a regular one and can be integrated explicitly. Indeed, $\varepsilon$ appears in the result of integral over each sub-triangle and vanishes after summing three integrals.

Let us consider $\Upsilon$ as the plane that contains element $E$. It is interesting to notice that this analytical scheme is also valid for the case in which the collocation point $\underline{x}_{c}$ is on plane $\Upsilon$ and is not in the element $E$ of type $O$ (Fig. 6b). This procedure provides a fast numerical calculation for evaluating the effective permeability of fractured porous media as demonstrated in section $\mathbf{0}$.

\section{Semi-analytical scheme}

Singular elementary integrals cannot be computed explicitly over the element of types 1 and 2 due to complicated nature of interpolation function in equations (49), (50). A semi-analytical procedure is developed to evaluate these kinds of integrals, which is based on the transformation to polar coordinate system centred at collocation point $\underline{x}_{c}$ such as one presented in the aforementioned scheme. In view of singularity treatment, the integration of variable $\rho$ is performed analytically. In this manipulation, the singularity of $\rho$ is disappeared. And then, the integration of variable $\theta$ is regular and can be integrated by Gaussian quadrature in one-dimensional.

In addition, the nearly singular elementary integral is also computed by this semianalytical procedure in which the polar coordinate system is centred at the projection $\underline{x}_{p c}$ of collocation point $\underline{x}_{c}$ on element $E$ (Fig. 6c). Supposing $h$ as the distance between $\underline{x}_{c}$ and element $E$, integral (14) for element of type 0 in polar coordinate reads: 


$$
I_{m k}=\sum_{k=1}^{3} p_{k}^{m} \underline{\zeta}_{k}\left(\int_{0}^{\varepsilon} \frac{\rho^{2} d \rho}{\left(\rho^{2}+h^{2}\right)^{3 / 2}} \int_{0}^{2 \pi} \underline{\phi} d \theta+\sum_{l=1}^{3} \int_{\theta_{l 1}}^{\theta_{l 2}} \underline{\phi} d \theta \int_{\varepsilon}^{\rho_{l}(\theta)} \frac{\rho^{2} d \rho}{\left(\rho^{2}+h^{2}\right)^{3 / 2}}\right)
$$

(a)

(b)
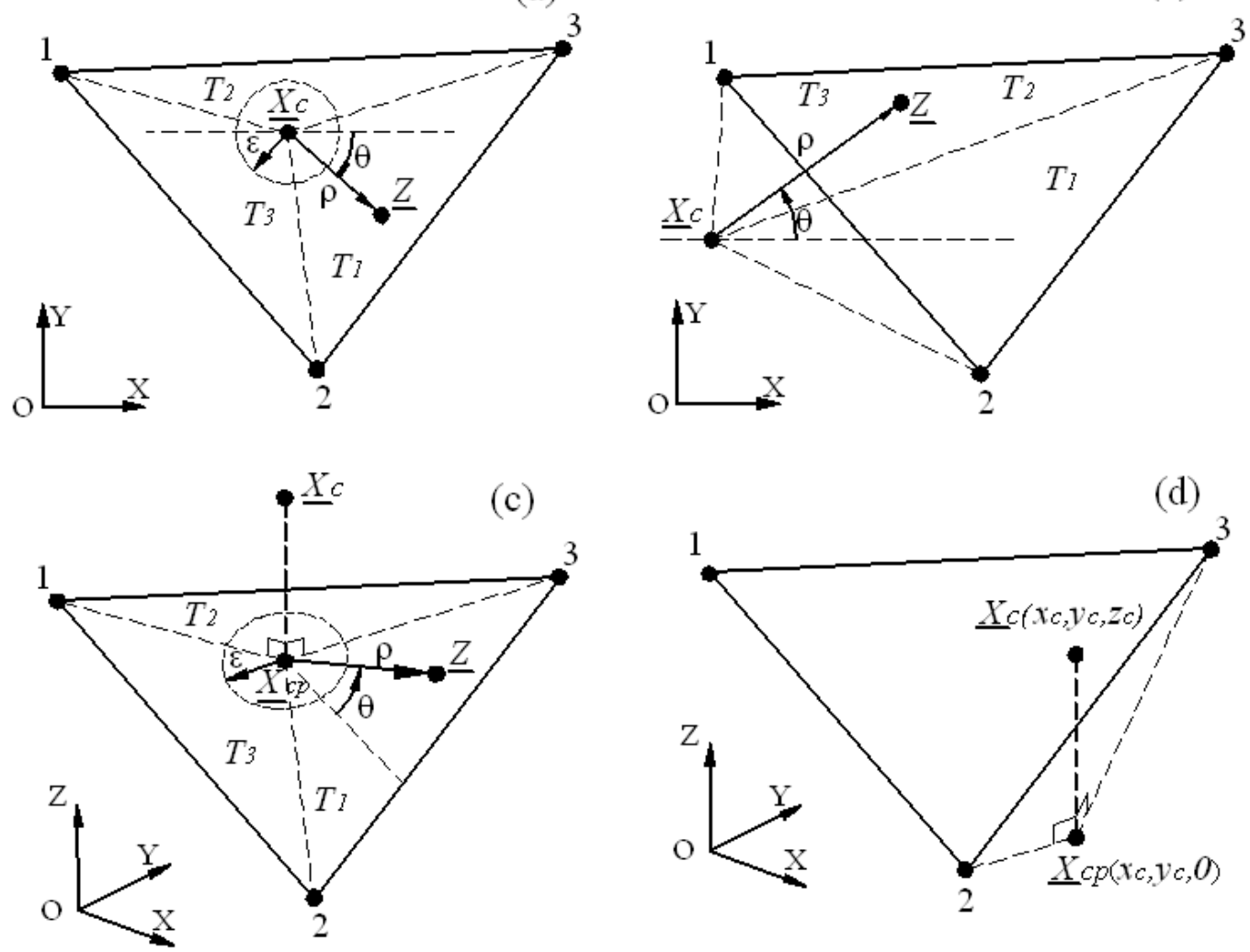

(d)

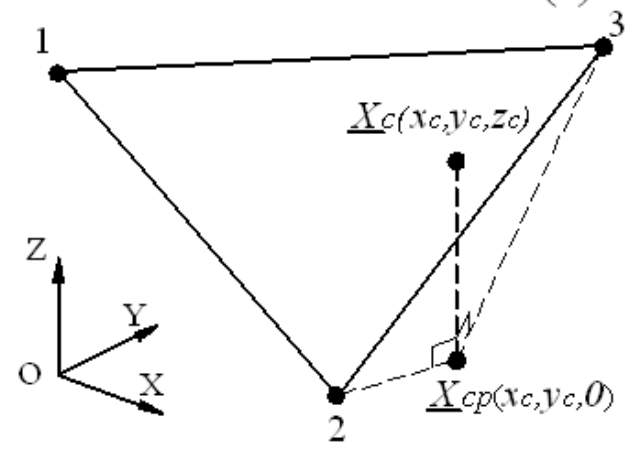

Fig. 6 Relative position between collocation point $\underline{x}_{c}$ and element $E$ ( $\Upsilon$ is supposed be $O x y$ ): (a) $\underline{x}_{\mathrm{c}}$ in element $E$; (b) is on plane $\Upsilon$ and not in the element $E$; (c) $\underline{x}_{\mathrm{c}}$ is not on $\Upsilon$ and $\underline{x}_{\mathrm{cp}}$ in element $E$; (d) $\underline{x}_{\mathrm{c}}$ is not on $\Upsilon$ and xcp not in element $E$.

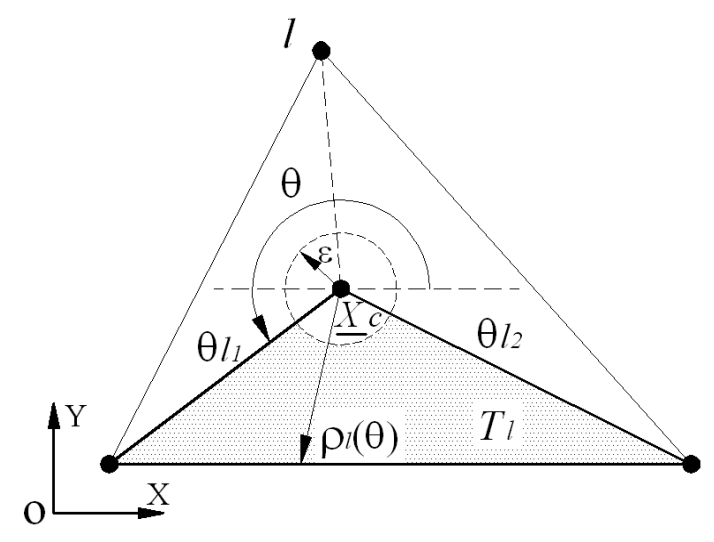

Fig. 7 Parameters of each sub-triangle $T_{l}$

As analysed in the analytical scheme in equation (17), only the second integral in this formula contributes to final result in which the integration of $\rho$ can be manipulated explicitly and then a numerical integration is employed for variable $\theta$. Unlike the general numerical procedure of boundary element method, the nearly singular integral is 
evaluated by some transformation technique for concentrating the Gauss's points in the vicinity of $\underline{x}_{p c}$ that costs many computational time and memory of PC.

The importance of correct evaluation of singular and nearly singular elementary integral takes full advantage for the good conditioning of linear system. Especially, the application of this numerical method in fractured porous rock formation in which the ratio between fracture conductivity and permeability of rock is normally considerable.

Numerical scheme

Let us consider $\underline{x}_{c p}$ as the projection of $\underline{x}_{c}$ on $\Upsilon$. Gaussian quadrature rule is an efficient method for computing the elementary integral in the case $\underline{x}_{c}$ is not on the plane $\Upsilon$ and $\underline{x}_{c p}$ is not in $E$ (Fig. 6d). Referring to the theory of numerical integration [43], the approximate value of integral (14) reads:

$$
I_{m}=\sum_{k=1}^{3} p_{k}^{m} \sum_{j=1}^{N g} w_{j} \frac{\nabla\left(\psi_{k}\left(\underline{x}_{G j}\right)\right) \cdot\left(\underline{x}_{c}^{i}-\underline{x}_{G j}\right)}{\left\|\underline{x}_{c}^{i}-\underline{x}_{G j}\right\|^{3}}
$$

where, $N g$ is the number of Gaussian point, $\underline{x}_{G j}$ the Gaussian point and $w_{j}$ weight of number $j$. This scheme can be also used to evaluate regular elementary integrals over the elements of type 1 and 2 where the collocation point $\underline{x}_{c}$ is on the plane $\Upsilon$

\section{VALIDATION OF NUMERICAL SOLUTION}

The limit case of a superconductive fracture is used as a benchmark problem for the numerical method in this work due to the fact that the closed-form solutions are well known for this reference case. In this view, two test-cases with different geometries are proposed.

\subsection{Single superconductive disc fracture}

The first case, an isolated superconductive fracture $(c \rightarrow \infty)$ is modelled by a zerothickness disc of radius $R$ in an infinite porous matrix under the limit condition $p_{\infty}(\underline{x})=x$. This disc is placed on the plane $X Y$ as depicted in Fig. 8. Pouya and Vu [20] presented a rigorous procedure to derive the explicit solution for a superconductive fracture (i.e. the pressure is constant in the fracture surface). The analytical solution of flux in the fracture disc is expressed in equation (20) and its contour is shown in Fig. 9.

Let us now apply the numerical calculation for a single superconductive fracture (c $>1$ for numerical modelling). The numerical solution of pressure obtained by singular integral equation is constant in superconductive fracture. Regarding the flux solution, a perfect agreement between analytical solution and numerical solution is also observed (Fig. 10). A relative error is then calculated to compare numerical and analytical results (21), where, $q_{\mathrm{n}}, q_{\mathrm{a}}$ are infiltration solutions obtained by numerical and analytical ways, respectively. The maximum value for the error is $E_{\mathrm{rmax}}\left(q_{\mathrm{x}}\right)=0,06 \%$.

$$
\underline{q}(\underline{\xi})=\frac{-8 k}{3 \pi r^{2}}\left\{\left(R r^{2}-R^{3}+{\sqrt{R^{2}-r^{2}}}^{3}\right) \boldsymbol{\delta}+\left(3 r^{2} \sqrt{R^{2}-r^{2}}+2 R^{3}-2 \sqrt{R^{2}-r^{2}}\right)(\underline{\hat{\xi}} \otimes \underline{\hat{\xi}})\right\} \boldsymbol{T} \underline{A}
$$

where, $r=\sqrt{\underline{\xi} . \underline{\xi}}, \underline{\xi}=\frac{1}{r} \underline{\xi}, \boldsymbol{T}=\boldsymbol{\delta}-\underline{e}_{\mathrm{z}} \otimes \underline{e}_{\mathrm{z}}$. 


$$
E_{r}=\left|\frac{q_{n}-q_{a}}{q_{a}}\right|
$$
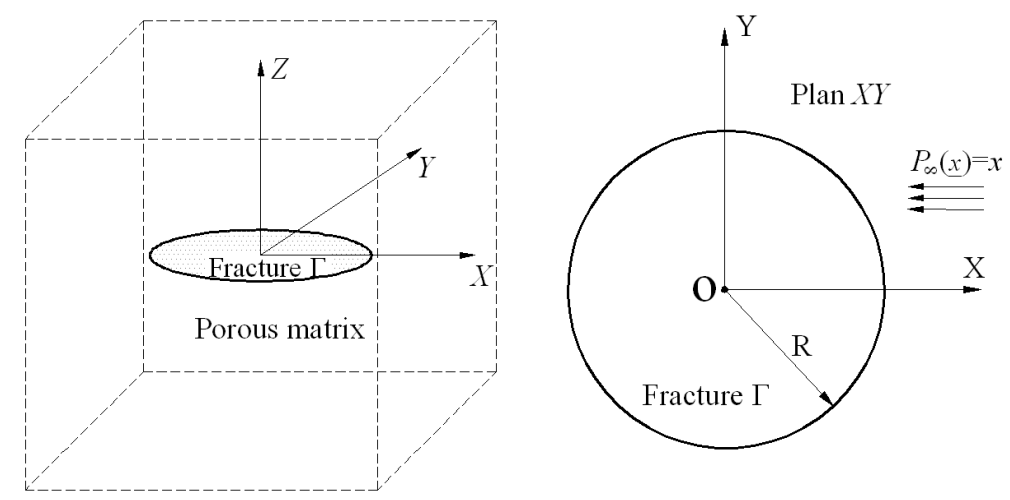

Fig. 8 An isolated superconductive disc in an infinite porous medium
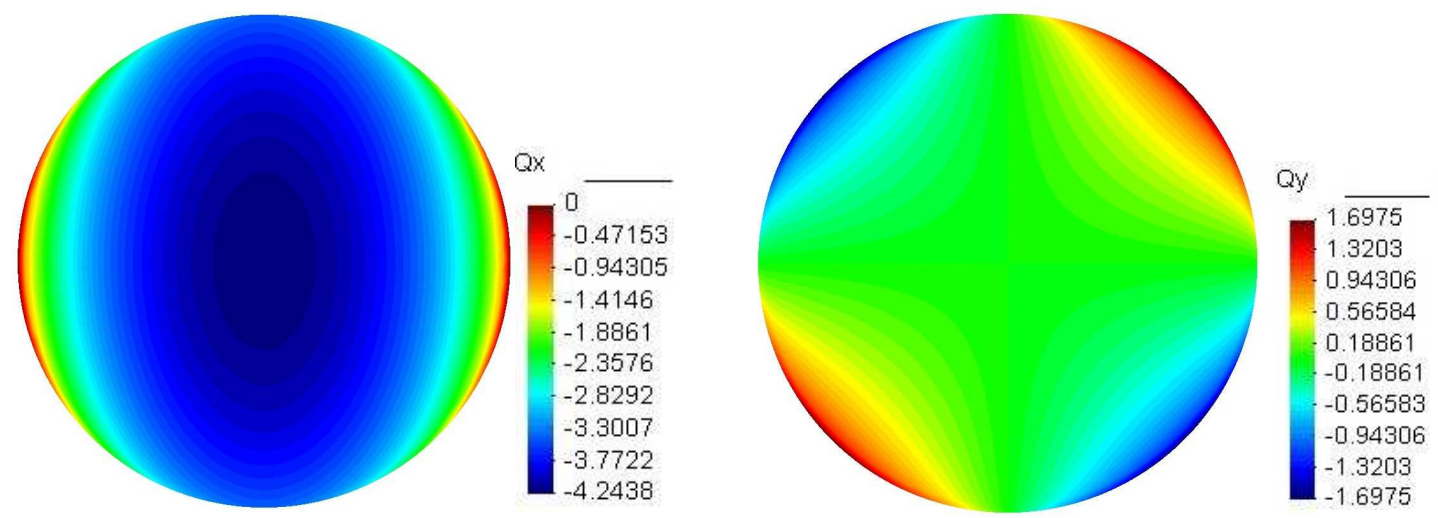

Fig. 9 Closed-form infiltration solution through a single superconductive circular fracture in an infinite matrix under a far-field gradient pressure $\underline{A}(1,0,0)[20]$

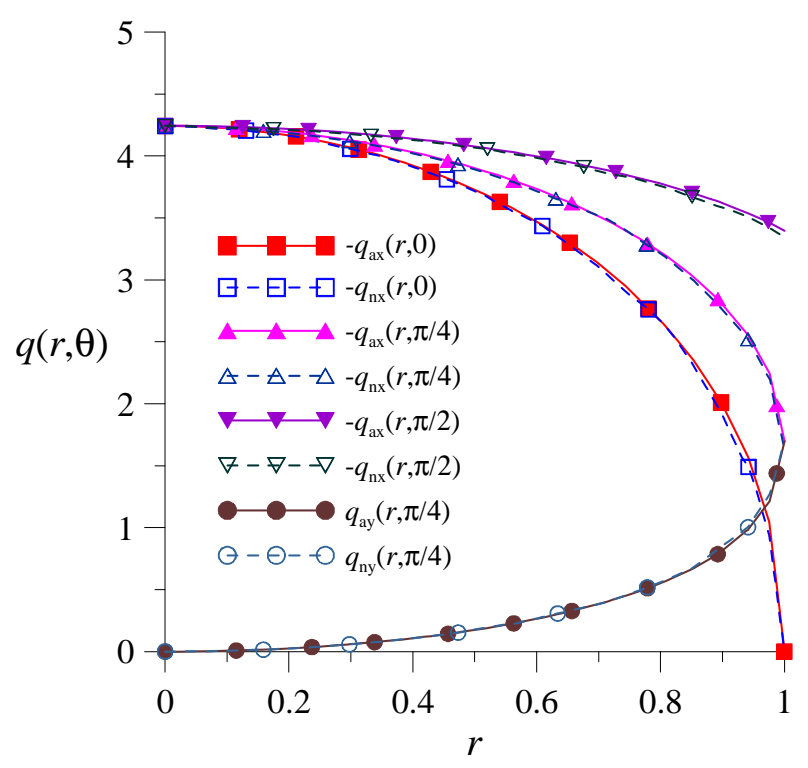

Fig. 10 Analytical vs. numerical solutions of infiltration $q(r)$ for different value of $\theta .0 \leq r \leq R$ and $q_{\mathrm{ax}}, q_{\mathrm{ay}}, q_{\mathrm{nx}}, q_{\mathrm{ny}}$ are analytical and numerical infiltration field on the fracture in $x$ and $y$ directions 


\subsection{Single superconductive band fracture}

In the second case, a single superconductive fracture $(c \rightarrow \infty)$ is modelled by a zerothickness band of finite width $L$ and of infinite length $\Lambda$ (numerically $\Lambda>>L$ ) in a $3 D$ infinite porous medium of isotropic permeability $\boldsymbol{k}=k \boldsymbol{\delta}$ (Fig. 11 left). Pressure field $p_{\infty}(\underline{x})=y$ is imposed at infinity. Theoretically, the fluid flow in every plane being perpendicular to $x$-axis is similar. As a consequence, this $3 \mathrm{D}$ configuration is equivalent to the fluid flow in an infinite plane (plane $Y Z$ ) containing an isolated fracture line of length $\mathrm{L}$ under the limit condition $p_{\infty}(\underline{x})=y$ (as seen in Fig. 11 right). For this $2 D$ configuration, a closed-form solution of flux in fracture line is given by Pouya and Ghabezloo [22]. From a numerical point of view, a band fracture with $\Lambda / L=5$ is modelled. The numerical solution of pressure field in fracture allows computing the flux in $y$-direction that passes at the middle of fracture. Good agreement between the current approach and the benchmark solutions is observed in Fig. 12.

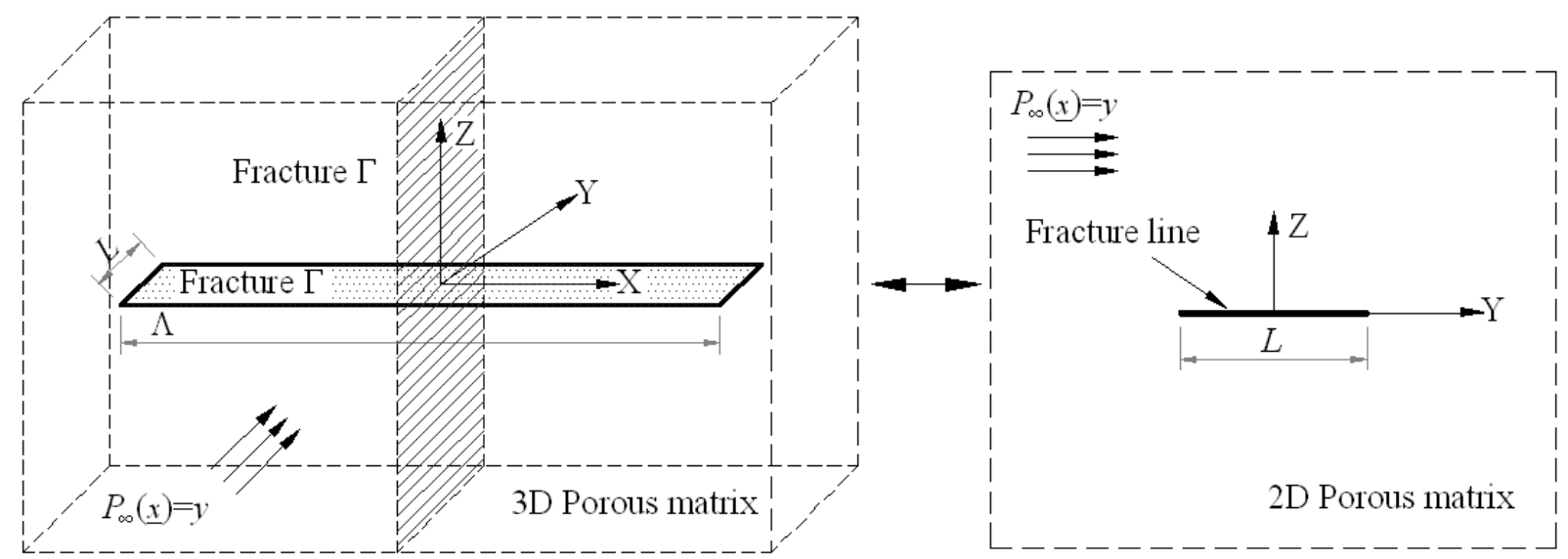

Fig. 11 Studied 3D band fracture and equivalent 2D configuration

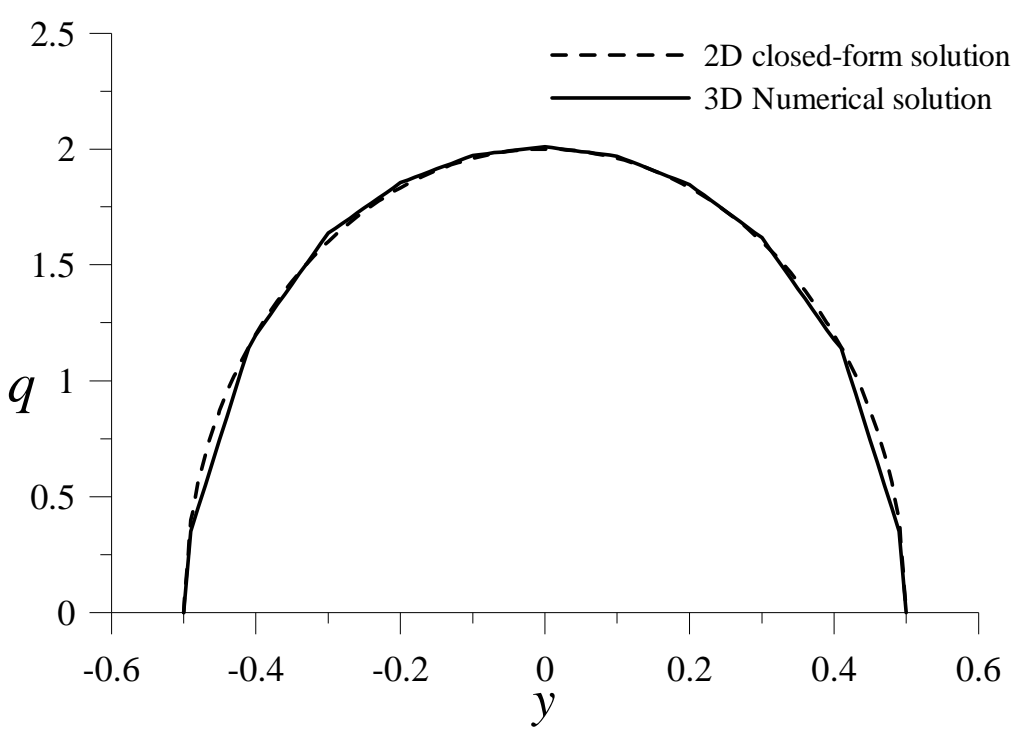

Fig. 12 Comparison of numerical solution in 3D configuration and closed-form solution in 2D configuration

These two examples of validation show that the developed numerical procedure provides results with high fidelity and accuracy. 


\section{APPLICATION TO FLOW THROUGH THREE-DIMENSIONAL FRACTURED POROUS MEDIA}

In order to demonstrate the capacity of the developed numerical procedure to simulate the flow through fracture networks, two application examples are presented in this section. The first one consists in modelling fluid flow through several intersecting circular fractures. In the second example, a curved fracture surface with a constant conductivity embedded in an infinite matrix is considered. In the first case, a consistent mesh is generated on the circular fractures as observed in Fig. 13. Fig. 14 presents the obtained pressure field in the fracture network. As observed, the pressure seems to be continuous on the intersection lines between fractures. The masse balance (7) at these lines can be verified and is well satisfied as it was also checked in our previous work on $2 \mathrm{D}$ problem [23]. In the second one, a curved fracture surface is modelled as a piece of a spherical surface (Fig. 15a). The numerical solution of pressure on this fracture is illustrated in Fig. 15b. However, we do not intend to analyse these results since it is not the main objective of this paper and we hope return to this subject in an application work.

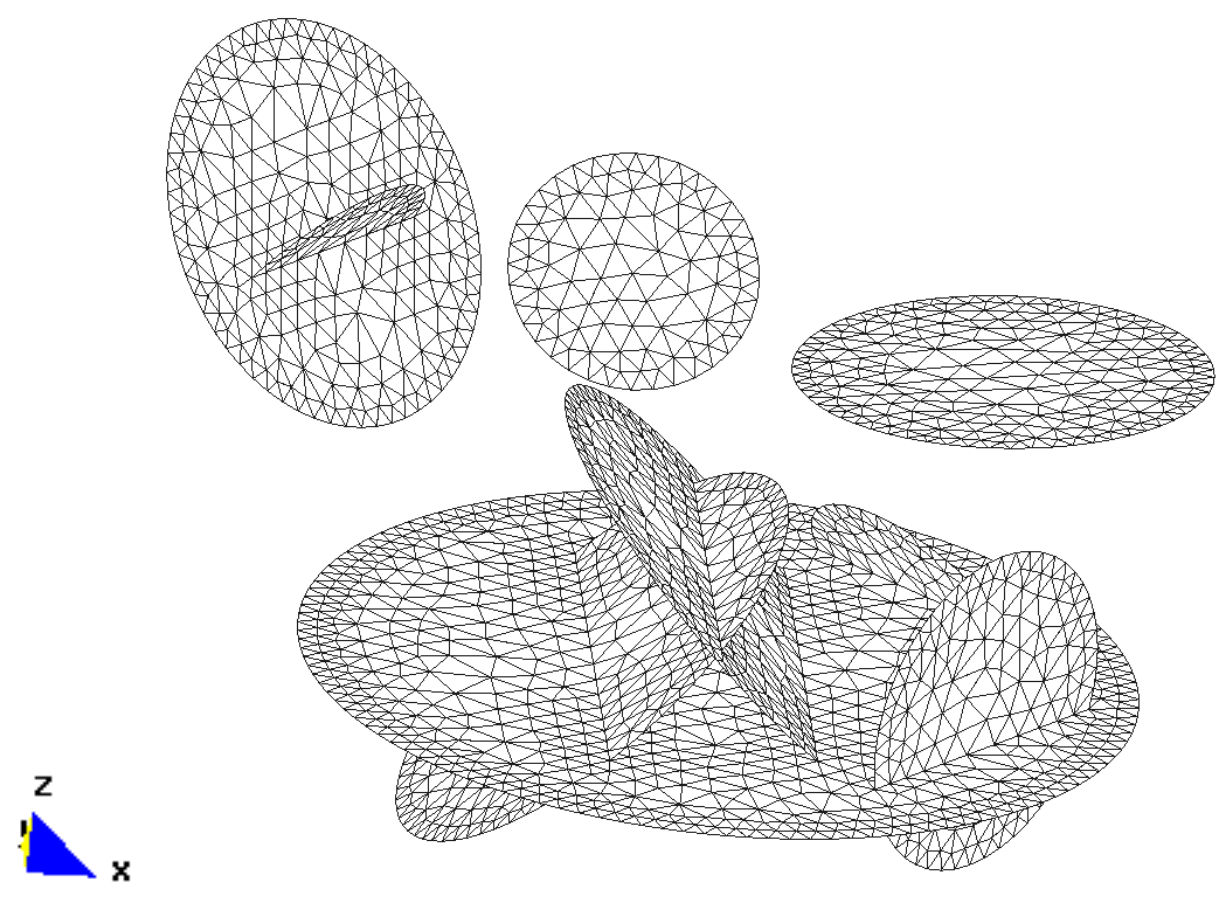

Fig. 13 Mesh of several intersecting fractures in an infinite isotropic matrix under a far-field pressure gradient $\underline{A}=(1,0,0)$ 


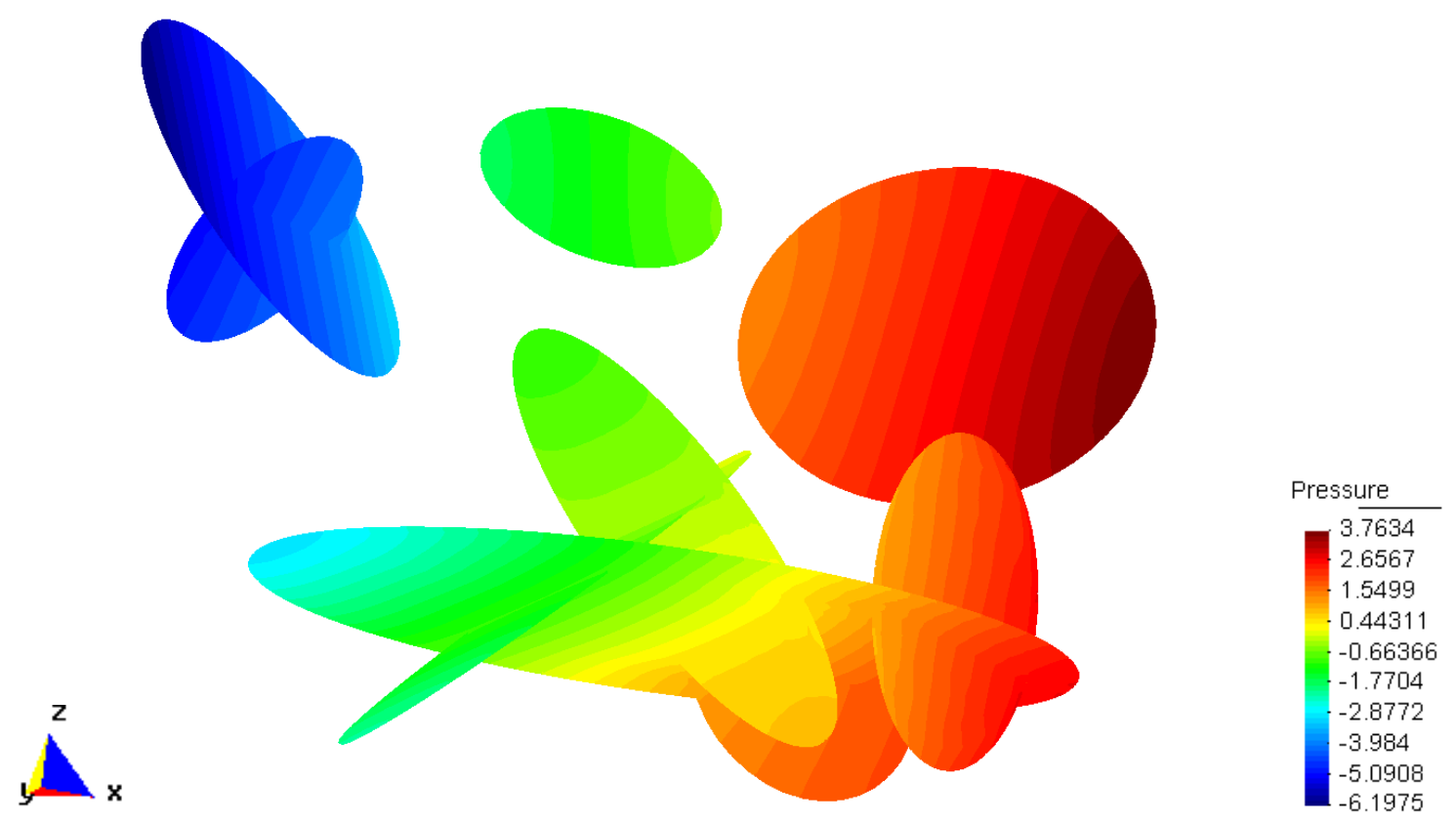

Fig. 14 Numerical solution of pressure field on several intersecting fractures in an infinite isotropic matrix under the a far-field pressure gradient $\underline{A}=(1,0,0)$

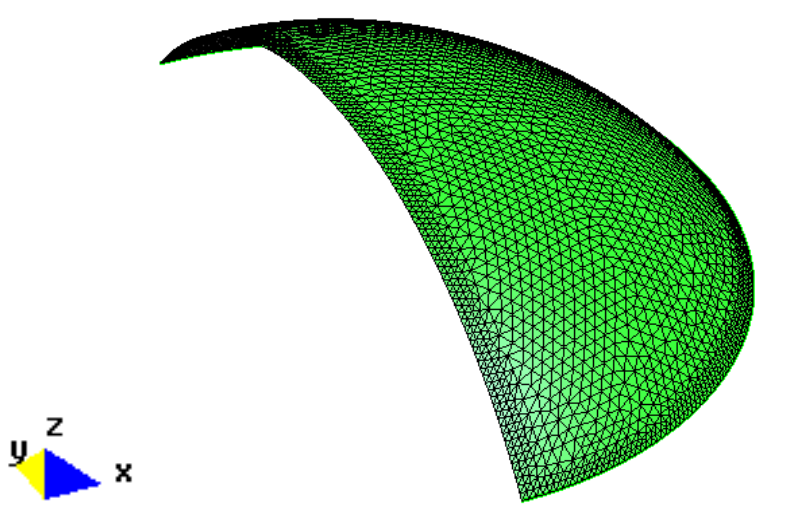

(a)

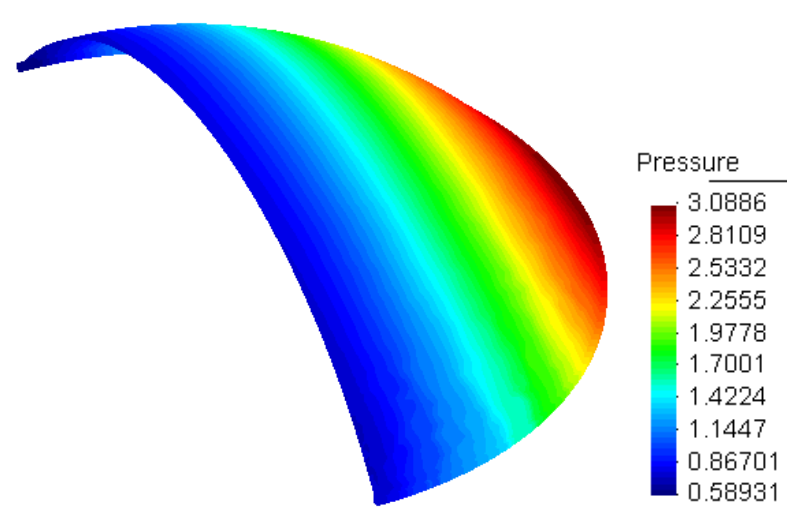

(b)

Fig. 15 Generated mesh (a) and numerical solution of pressure field (b) on a curved fracture surface embedded in an infinite isotropic matrix under a uniform pressure gradient $\underline{A}=(1,0,0)$

\section{APPLICATION TO EFFECTIVE PERMEABILITY ESTIMATION}

Contrary to the case of superconductive disc fracture, the case of disc fracture having a finite conductivity has not an analytical solution. Numerical computation based on singular integral method can deal with this problem. The numerical procedure described in section 3 is applied for a single circular fracture with different conductivity values. Obtained numerical solutions allow us to determine a semi-analytical solution of infiltration through this fracture and effective permeability of fractured porous media as a function of fracture conductivity, matrix permeability and geometry variables.

\subsection{Semi-analytical solution of flux on fracture}

An infinite body with isotropic permeability $\boldsymbol{k}=k \boldsymbol{\delta}$ containing a single fracture disc of 
radius $R$ and of constant conductivity $c$ is considered (Fig. 8). The body is submitted at infinity to a constant gradient $\underline{A}$. Equation (8) is written for this problem such as:

$$
p(\underline{x})=\underline{A} \cdot \underline{x}-\frac{c}{4 \pi k} \int_{\Gamma} \frac{\nabla p(\underline{z}) \cdot(\underline{x}-\underline{z})}{\|\underline{x}-\underline{z}\|^{3}} d z
$$

Let us introduce following dimensionless variables:

$$
\underline{X}=\frac{\underline{x}}{R}, \lambda=\frac{c}{4 \pi k R}
$$

And the notation,

$$
P_{\lambda}(\underline{X})=\frac{p(\underline{x})}{R|\underline{A}|}, \quad \nabla p(\underline{x})=|\underline{A}| \underline{\nabla} P_{\lambda}(\underline{X})
$$

By supposing $\Gamma^{*}$ as a circle of unit radius centred at the origin of coordinates system, equation (22) reads:

$$
P_{\lambda}(\underline{X})=\frac{\underline{A}}{|\underline{A}|} \cdot \underline{X}-\lambda \int_{\Gamma^{*}} \frac{\nabla P_{\lambda}(\underline{X}) \cdot(\underline{X}-\underline{Z})}{\|\underline{X}-\underline{Z}\|^{3}} d Z
$$

Numerical procedure described in section 3 is employed to resolve equation (25) for calculating the pressure field $P_{\lambda}(\underline{X})$ corresponding to different values of $\lambda$. The numerical result for $\lambda=2$ is shown in Fig. 16. Evaluation of integral flux field over the fracture surface gives:

$$
\underline{Q}(\underline{x})=-c \int_{\Gamma} \nabla p(\underline{x}) d x=-c R^{2}|\underline{A}| \int_{\Gamma^{*}} \nabla P_{\lambda}(\underline{X}) d X
$$

By defining,

$$
Q^{*}(\underline{X})=\frac{\underline{Q}(\underline{x}) \cdot \underline{A}}{R^{2}|\underline{A}|^{2}}=-c \underline{A} \cdot \int_{\Gamma^{*}} \nabla P_{\lambda}(\underline{X}) d X
$$
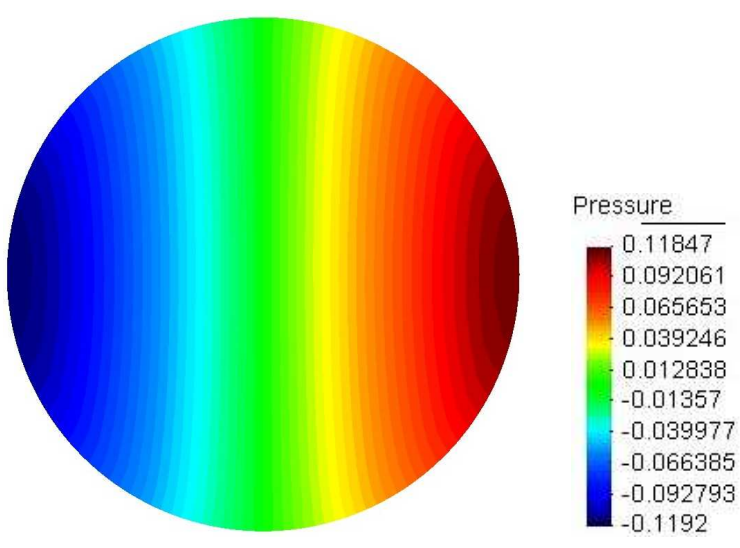

(a)

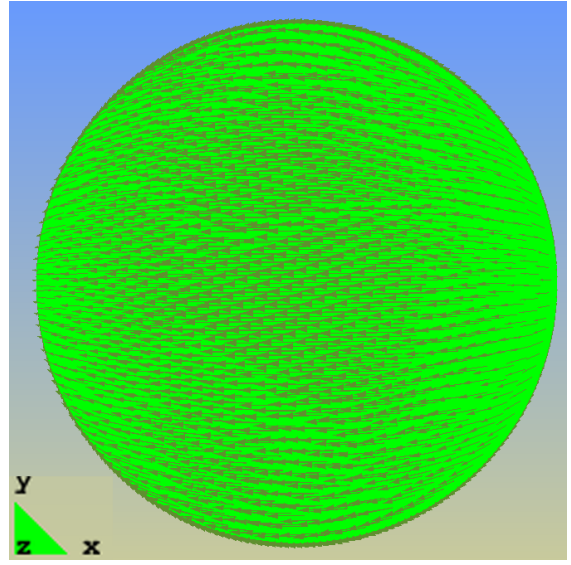

(b)

Fig. 16 Pressure field in the fracture $(\lambda=2)$

The value obtained numerically for $P_{\lambda}(\underline{X})$ allows describing with a great precision the 
relation between two variables $Q^{*}(\underline{X})$ and $\lambda$ by the following formulae as plotted in Fig. 17.

$$
\frac{c}{Q^{*}}=\frac{3 \pi}{4} \lambda+\frac{1}{3}
$$

Despite the fact that the above expression is obtained numerically by curve fitting, it reveals to be extremely accurate. Equation (28) provides a relationship between integral of flux field over the circular disc fracture $\underline{Q}(\underline{x})$ and the problem parameters, namely: fracture conductivity $c$, matrix permeability $k$, and fracture geometry $R$ :

$$
\underline{Q}(\underline{x})=-\frac{16 k c R^{3}}{3 c+\frac{16}{3} k R} \underline{A}
$$

This equation can be verified by its asymptotic behaviour when $\lambda \rightarrow \infty$ such: $\lim _{c \rightarrow \infty} \underline{Q}(\underline{x})=-\frac{16 k R^{3}}{3} \underline{A}$, which is according to close-formed solution for superconductive circular disc fracture given by Pouya and $\mathrm{Vu}$ [20]. Furthermore, this result is consistent with one obtained by Shafiro and Kachanov [17] for the superconductive flattened ellipsoidal inclusion with Darcy's flow. This property reveals a high precision of the semi-analytical solution (29). Therefore, this result can be used confidently in the following to calculate the effective permeability of a fractured porous media.

In order to determine the equivalent permeability tensor of fractured porous media, equation (29) could be expressed for the general case of an arbitrary circular fracture in space such as:

$$
\underline{Q}(\underline{x})=-\frac{16 k c R^{3}}{3 c+\frac{16}{3} k R} \boldsymbol{T} \cdot \underline{A}
$$

where, $\boldsymbol{T}$ is the projection operator on the plane of fracture given by the following expression in which $\underline{n}$ represents the unit normal of the fracture plane:

$$
\boldsymbol{T}=\boldsymbol{\delta}-\underline{n} \otimes \underline{n}
$$

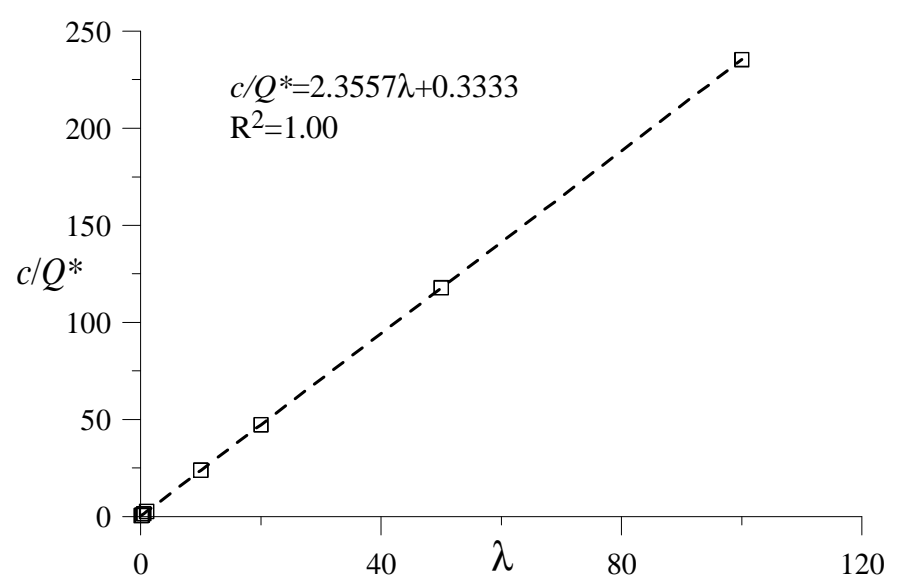

Fig. 17 Numerical solution for $\mathrm{c} / Q^{*}(\underline{X})$ depending on $\lambda$, fitted by a linear function 


\subsection{Upscaling of effective permeability}

Let us consider a set of fractures $\Gamma^{m}$ embedded in a reference homogeneous volume $\Omega$ of an infinite $3 \mathrm{D}$ porous medium having isotropic permeability $\boldsymbol{k}=k \boldsymbol{\delta}$. By considering the fracture as the limit case of a thin permeable layer, the average velocity in $\Omega$ reads $[13,22,16,20]$ :

$$
\underline{V} \equiv \frac{1}{\Omega}\left[\int_{\Omega} \underline{v} d \Omega+\sum_{m} \int_{\Gamma^{m}} \underline{q} d \Gamma\right]
$$

A linear pressure $p(\underline{x})=\underline{A} \cdot \underline{x}$ is applied at the boundary $\partial \Omega$. Using the divergence theorem for the pressure function in a domain containing des discontinuities with the fact that $\llbracket p \rrbracket=0$ on the fractures, allows the determination of the average pressure gradient as follows:

$$
\underline{G} \equiv \frac{1}{\Omega} \int_{\Omega} \nabla p d \Omega=\underline{A}
$$

Introducing (1) into the first term of $\underline{V}$ in (32) yields:

$$
\frac{1}{\Omega} \int_{\Omega} \underline{v} d \Omega=-\boldsymbol{k} \cdot \underline{A}
$$

In addition, the effective permeability $\hat{\boldsymbol{k}}$ is yielded by $\underline{V}=-\hat{\boldsymbol{k}} \cdot \underline{G}$, hence,

$$
\hat{\boldsymbol{k}}=\boldsymbol{k}+\boldsymbol{k}^{f}
$$

where, $\boldsymbol{k}^{f}$ is the contribution of fractures to the effective permeability defined as:

$$
\frac{1}{\Omega} \sum_{m} \int_{\Gamma^{m}} \underline{q} d \Gamma=-\boldsymbol{k}^{f} \cdot \underline{A}
$$

According to the above-mentioned analyses, the semi-analytical solution expressed in equation (30) could be used to calculate the effective permeability in equation (35) when the domain $\Omega$ tends to infinity. For a weak fracture density, dilute Mori-Tanaka scheme for estimation of $\hat{\boldsymbol{k}}$ can be used. In this scheme, the interaction between fractures is neglected and each fracture is subject to the same far-field pressure, unperturbed by the presence of other fractures. In this approximation, the effective permeability tensor $\hat{\boldsymbol{k}}$ is expressed as the sum of the matrix permeability and additional permeability due to each fracture $\boldsymbol{k}^{f}$ :

$$
\hat{\boldsymbol{k}}=k \boldsymbol{\delta}+\sum_{m} \boldsymbol{k}_{m}^{f}
$$

Assuming that each family of fractures $\Gamma^{m}$ is characterized by a mean radius $R^{m}$, an orientation $\underline{n}^{m}$ (unit normal of fracture), a conductivity $c^{m}$ and a density (number of fracture center per unit volume) $\rho^{m}$, the expression of $\boldsymbol{k}_{m}^{f}$ will be obtained as follows:

$$
\boldsymbol{k}_{m}^{f}=\rho^{m} \frac{16 k c^{m} R^{m 3}}{3 c^{m}+\frac{16}{3} k R^{m}}\left(\boldsymbol{\delta}-\underline{n}^{m} \otimes \underline{n}^{m}\right) \cdot \underline{A}
$$


Equations (37)-(38) cover arbitrary orientations of fractures and constitute an approach to calculate fluid flow in porous media with stochastic distribution of fracture.

For the case of random orientation of fractures (overall isotropy), the average value of $\underline{n} \otimes \underline{n}$ is $\frac{1}{3} \boldsymbol{\delta}$ and the effective permeability tensor is isotropic and reads $\hat{\boldsymbol{k}}=\hat{k} \boldsymbol{\delta}$. If all fractures with density $\rho$ have identical shapes (i.e. identical radius $R$ ) and identical conductivity $c$, Mori-Tanaka's estimate results in an isotropic effective permeability:

$$
k^{M T}=k+\rho \frac{32 k c R^{3}}{9 c+16 k R}
$$

Self-consistent scheme consists in replacing the matrix permeability $k$ in the fracture contribution $\boldsymbol{k}^{f}$ (second term in the right hand side of equation (39)) by the unknown effective permeability $\hat{k}=k^{s c}$. This leads the following second degree equation for $k^{s c}$.

$$
k^{s c}=k+\rho \frac{32 k^{s c} c R^{3}}{9 c+16 k^{s c} R}
$$

Indeed, the dilute Mori-Tanaka's estimate (39) corresponds to the first order development of Equation (40) with respect to $\rho$.

In order to unify the results for other fracture shapes, it is useful to use dimensionless variables. We recall the definition of the excluded volume $V_{e x}$ to define the dimensionless density. The excluded volume for a fracture is defined as the volume into which the center of another fractures may not enter if there is no fracture intersection. Therefore, the dimensionless density $\bar{\rho}$ that is defined as the number of fractures per excluded volume reads [45]:

$$
\bar{\rho}=\rho V_{e x}
$$

where, $V_{e x}=\pi^{2} R^{3}$ for disc fracture of radius $R$ [46]. In order to study the variation of effective permeability versus $\bar{\rho}$, another dimensionless variable is introduced as follows:

$$
\bar{\lambda}=\frac{c}{4 \pi \hat{k} R}
$$

Fig. 18 displays the variation of self-consistent estimate $k^{s c}$ with the fracture density for different values of fracture conductivity. In the limit case $\lambda \rightarrow \infty$, the self-consistent model (40) reveals a critical value $\bar{\rho}_{c} \approx \frac{9 \pi^{2}}{32}$ i.e $\rho \approx \frac{9}{32 R^{3}}$ which is exactly the singular value of density obtained by Pouya and $\mathrm{Vu}$ [20] for the case of superconductive circular fracture (i.e., $c \rightarrow \infty$ ). This result can have another interpretation: The limit case of $\lambda \rightarrow \infty$ corresponds to an impermeable matrix $(k \rightarrow 0)$. For this case, the effective permeability can be different from zero beyond the critical value $\bar{\rho}_{c}$. According to Dormieux and Kondo [18], this critical density could be considered as the percolation threshold for the fracture network.

As it can be observed in Fig. 18, the variation of $\bar{\lambda}$ versus $\bar{\rho}$, for the case $k=0$ and $\bar{\rho} \geq \bar{\rho}_{c}$, can be approximated by a line with the following equation: 


$$
\bar{\rho} \geq \bar{\rho}_{c} ; \quad \frac{1}{\bar{\lambda}}=2.55\left(\bar{\rho}-\frac{9 \pi^{2}}{32}\right)
$$

Furthermore,

$$
\bar{\rho} \geq \bar{\rho}_{c} ; \quad \frac{k^{s c}}{k}=2.55 \lambda\left(\bar{\rho}-\frac{9 \pi^{2}}{32}\right)
$$

For a finite value of $\lambda$, the slope of curves $1 / \bar{\lambda}$ increases with $\bar{\rho}$ up to an asymptotic value. It also exits a critical value for each curve beyond which the effective permeability increases up to one order of magnitude. This critical point can be determined as the intersection of the initial and final tangents of the curve.

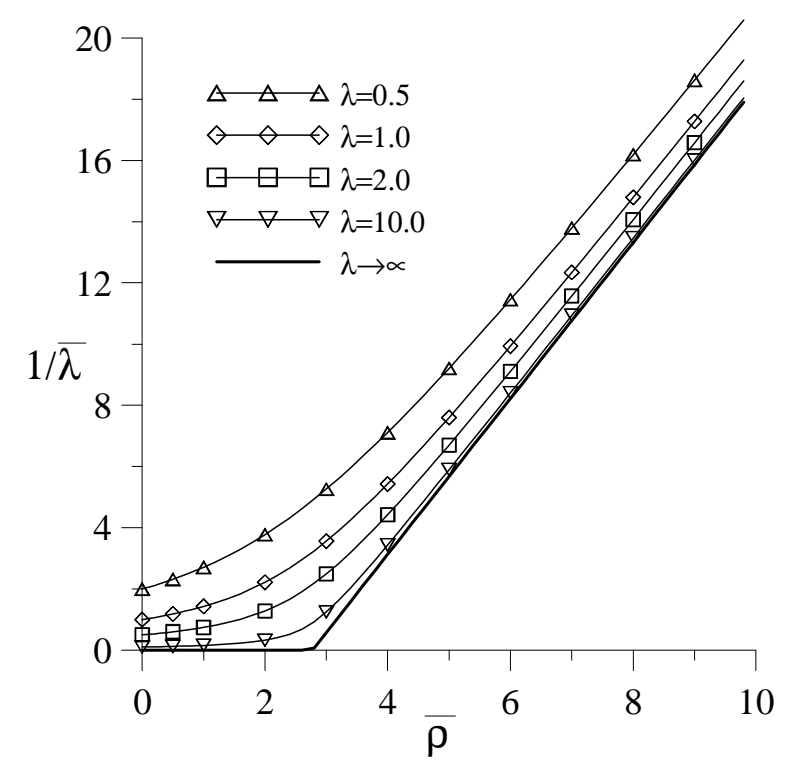

Fig. 18 Self-consistent scheme estimation of dimensionless variable $1 / \bar{\lambda}$ plotted as a function of the dimensionless density for different values of $\lambda$

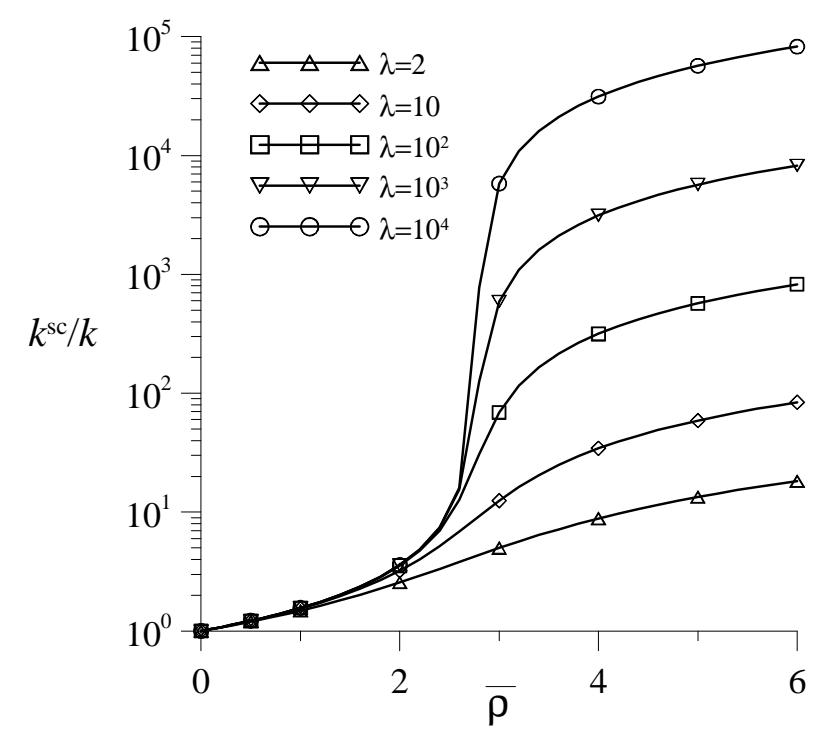

Fig. 19 Variation of $k^{\text {sc }} / k$ versus dimensionless density for different values of $\lambda$ 
Furthermore, the variation of $k^{\mathrm{sc}} / k$ as function of dimensionless density $\bar{\rho}$ is plotted in Fig. 19 for different value of $\lambda$. The critical value $\bar{\rho}_{c}$ is also observed in this figure for great values of $\lambda$ corresponding to a jump in $k^{\text {sc }}$. The calculated $\bar{\rho}_{c}$ is very close to the value obtained by Huseby et al. [47].

As mentioned earlier, the effective permeability of a fractured porous medium can be evaluated by considering only a single fracture. Hence, the computation can be carried out by employing only analytical and semi-analytical elementary integrals in numerical procedure since all collocation points are located on the fracture plane. This leads to a fast and efficient numerical programme for computing equivalent permeability of a fractured material.

\section{CONCLUSION}

Numerical computation based on singular integral equations method is described to resolve the general potential solution presented by Pouya [24] for the fluid flow in an infinite fractured porous media under a far-field pressure gradient. In this framework, a consistent mesh is only required on the surface of fractures of the studied network. Numerical solution is successfully validated by comparing numerical results with analytical solutions of two benchmark problems, namely: a single superconductive circular disc-shaped fracture in $3 \mathrm{D}$ and a single superconductive fracture line in $2 \mathrm{D}$. A semi-analytical solution for infiltration through the fracture versus fracture conductivity, matrix permeability and fracture geometry parameters based on numerical solutions is obtained for a single fracture and different values of fracture conductivity. This is a key result to upscale the effective permeability of an infinite porous medium containing a distribution of fractures with finite conductivity.

The numerical procedure presented in this study is valid for intersecting curved fractures surface in various scales. Thus, it can be applied to model the flow in a set of curved faults under a far-field pressure condition. In particular, the developed model can be used for a more accurate simulation of hydromechanical behaviour of the "fault zones" where a dense fracture networks surround the central part of the fault [48]. This possibility opens a way to motivate further works on the industrial application fields as petroleum, geothermic, geological $\mathrm{CO}_{2}$ storage, etc.

It is worth reminding that only fractures with non-pressure jump between their two faces are considered in the present work. However, a fracture can act as an impermeable membrane with a pressure discontinuity across its surface. A closed-form solution for the potential discontinuity across an impervious disc embedded in an infinite matrix is proposed by Martin [49]. The study on 2D problem, in order to upscale the effective permeability of a porous media containing impervious crack lines, is an ongoing work and will be addressed in a future paper. 


\section{ACKNOWLEDGEMENTS}

The work presented in this paper is co-funded by BRGM through an "Institut Carnot" research fund and IFSTTAR. The authors also acknowledge the fruitful discussions with Dr Pierre Sochala.

\section{REFERENCES}

1. Long JCS, Gilmour P, Witherspoon PA. A model for steady fluid flow in random threedimensional networks of disk-shaped fractures. Water Resources Research 1985; 21(8):1105-1115.

2. Cacas MC, Ledoux E, De Marsily G. Modeling fracture flow with a stochastic discrete fracture network: calibration and validation. Water Resources Research 1990; 26(3): 479500 .

3. Pouya A, Fouché O. Permeability of 3D discontinuity networks. New tensors from boundary-conditioned homogenisation. Advances in Water Resources 2009; 32:303-314.

4. Jafari A, Babadagli T. Effective Fracture Network Permeability of Geothermal Reservoirs. Geothermics 2001; 40:25-38.

5. ITASCA Software. Universal Distinct Element Code (UDEC). http://www.itascacg.com/udec/

6. Beicip-Franlab. Fractured Reservoir Characterization and Modelling (FracaFlow).

7. Barenblatt GI, Zheltov YP, Kochina IN. Basic concepts in the theory of seepage of homogeneous liquids in fissured rocks. Sov. Appl. Math. Mech., Engl. Transl. 1960; 24:852-864.

8. Baca R, Arnett R, Langford D. Modeling fluid flow in fractured porous rock masses by finite element techniques. Int. J. Numer. Methods Fluids 1984; 4:337-348.

9. Ghabezloo S, Pouya A. Numerical upscaling of the permeability of a randomly cracked porous medium. The 12th international conference of International Association for Computer Methods and Advances in Geomechanics (IACMAG) 2008; Goa, India: 25362543.

10. Woodbury A, Zhang K. Lanczos method for the solution of groundwater flow in discretely fractured porous media. Advances in Water Resources 2001; 24:621-630.

11. Lesinigo M, D'Angelo C, Quarteroni A. A multiscale Darcy-Brinkman model for fluid flow in fractured porous media. Numerische Mathematik 2010; 1-36.

12. Castaing C, Genter A, Bourgine B, Chilès JP, Wendling J, Siegel P. Taking into account the complexity of natural fracture systems in reservoir single-phase flow modelling. Journal of Hydrology 2002; 266:83-98.

13. Bogdanov II, Mourzenko VV, Thovert JF, Adler PM. Effective permeability of fractured porous media in steady state flow. Water Resources Research 2003; 39(1):1-16.

14. Angot P, Boyer F, Hubert F. Asymptotic and numerical modelling of flows in fractured porous media. M2AN 2009; 43(2):239-275.

15. Grillo A, Logashenko D, Stichel, Wittum G. Simulation of density-driven flow in fractured porous media. Advances in Water Resources 2010; 33(12):1494-1507.

16. Mourzenko VV, Bogdanov II, Thovert JF, Adler PM. Three-dimensional numerical simulation of single-phase transient compressible flows and well-tests in fractured formations. Mathematics and Computers in Simulation 2010; 81(10):2270-2281.

17. Shafiro B, Kachanov M. Anisotropic effective conductivity of material with nonrandomly oriented inclusions of divers ellipsoidal shapes. Journal of applied physics 2000; 87(12):8561-8569.

18. Dormieux L, Kondo D. Approche micromécanique du couplage perméabilité endommagement. C.R. Mecanique 2004 ; 332:135-140. 
19. Barthélémy JF. Effective Permeability of Media with a Dense Network of Long and Micro Fractures. Transp Porous Med 2009; 76:153-178.

20. Pouya A, Vu MN. Flow around a disc-shaped crack inclusion in infinite matrix. Application to permeability upscaling of fractured porous media. Advances in Water Resources 2011; (accepted).

21. Liolios PA, Exadaktylos GE. A solution of steady-state fluid flow in multiply fractured isotropic porous media. International Journal of Solids and Structures 2006; 43:39603982.

22. Pouya A, Ghabezloo S. Flow around a crack in a porous matrix and related problems. Transport in Porous Media 2010; 84(2):511-532.

23. Pouya $\mathrm{A}, \mathrm{Vu} \mathrm{MN}$. Numerical modelling of steady-state flow in 2D cracked anisotropic porous media by singular integral equations method. Transport in Porous Media 2012; 93(3): 475-493.

24. Pouya A. Three-dimensional flow in fractured porous media: a potential solution based on singular integral Equation. Advances in Water Resources 2012; 42: 37-46.

25. Guéguen Y, Palciauskas V. Introduction to Physics of Rocks. Princeton Univ. Press 1994.

26. Zhou CB, Sharma RS, Chen YF, Rong G. Flow-stress coupled permeability tensor for fractured rock masses. International Journal for Numerical and Analytical Methods in Geomechanics 2008; 32(11):1289-1309.

27. Adler PM. Porous Media: Geometry and Transports. Butterworth-Heinemann: Woburn, Mass., 1992.

28. Aifantis EC. On Barrenblatt's problem. Int. J. Eng. Sci. 1980; 18:857- 867.

29. Brebbia CA, Dominguez J. Boundary Elements: An Introduction Course. McGraw-Hill: New York, 1989.

30. Brebbia CA, Telles J, Wrobel L. Boundary Element Techniques Theory and Applications in Engineering. Springer-Verlag: Berlin, 1984.

31. Bonnet M. Equations Intégrales et Éléments de Frontière. CNRS Editions/Eyrolles : Paris, 1995.

32. Muskhelishvili NI. Singular Integral Equations. P. Noordhoff Ltd.: Groningen, Holland, 1953.

33. Erdogan F. Approximate Solutions of Systems of Singular Integral Equations. SIAM Journal of Applied Mathematics 1969; 17:1041-1059.

34. Martin PA. End-point behaviour of solutions to hypersingular integral Equations. Proc. R. Soc. London A 1991; 432:301-320.

35. Wolfe P. Eigen/functions of the integral Equation for the potential of the charged disk. $J$. Math. Phys. 1971; 12:1215-1218.

36. Boersma J, Danicki E. On the solution of an integral Equation arising in potential problems for circular and elliptic disks. SIAM J. Appl. Math. 1993; 53:931-941.

37. Guiggiani M. The evaluation of Cauchy principal value integrals in the boundary element method-A review. Mathematical and Computer Modelling - Special Issue on BIEM/BEM, $1991 ; 15(3-5): 175-184$.

38. Bonnet M. Methodes des Equations Integrates Regularises en Elastodynamique. Ph.D. Thesis, Ecole National des Ponts et Chaussees, 1986.

39. Alok Sutradhar, Glaucio HP, Leonard JG. Symmetric Galerkin Boundary Element Method. Springer, 2008.

40. Guiggiani M, Gigante A. A general algorithm for multidimensional Cauchy principal value integrals in the boundary element method. J Appl Mech Trans ASME 1990; 57:906-915.

41. Karami G, Derakhshan D. An efficient method to evaluate hypersingular and supersingular integrals in boundary integral quations analysis. Engineering Analysis with Boundary Elements 1999; 23:317-326.

42. Liu YJ, Zhang D, Rizzo FJ. Nearly singular and hypersingular integrals in the boundary 
element method. In: Brebbia, C.A., Rencis, J.J. (Eds.), Boundary Elements XV. Computational Mechanics Publication:453-468, Southampton, 1993.

43. Davis JJ, Rabinowitz P. Methods of numerical integration. Academic Press, 1975.

44. Balberg I, Anderson $\mathrm{CH}$, Alexander S, Wagner N. Excluded volume and its relation to the onset of percolation. Phys. Rev. B 1984; 30(39):33-43.

45. Adler PM, Thovert JF. Fractures and Fracture Networks. Kluwer Acad.: Norwell, Mass., 1999.

46. Charlaix E, Guyon E, Rivier N. A criterion for percolation threshold in a random array of plates. Solid State Commun 1984; 50(11):999-1002.

47. Huseby O, Thovert JF, Adler PM, Geometry and topology of fracture systems, J. Phys. A 1997; 30:1415-1444.

48. Seyedi DM, Ducellier A, Vu MN, Pouya A, A two-scale model for simulating the hydromechanical behavior of faults during $\mathrm{CO} 2$ geological storage operation, $45^{\text {th }}$ US Rock Mechanics/Geomechanics Symposium 2011, San Fransisco, June 26-29.

49. Martin, PA, On potential flow past wrinkled discs. Proceedings of the Royal Society A 1998; 454: 2209-2221.

\section{APPENDIX A}

The shape functions of an arbitrary element are obtained from ones on the reference element. The equilateral triangle is selected as the reference element in the parameter space $\{\eta, \xi\}$ (Fig. 20) with the shape functions as:

$$
\psi_{1}(\eta, \xi)=\frac{\sqrt{3}(1-\eta)-\xi}{2 \sqrt{3}} ; \quad \psi_{2}(\eta, \xi)=\frac{\sqrt{3}(1+\eta)-\xi}{2 \sqrt{3}} ; \quad \psi_{3}(\eta, \xi)=\frac{\xi}{\sqrt{3}}
$$

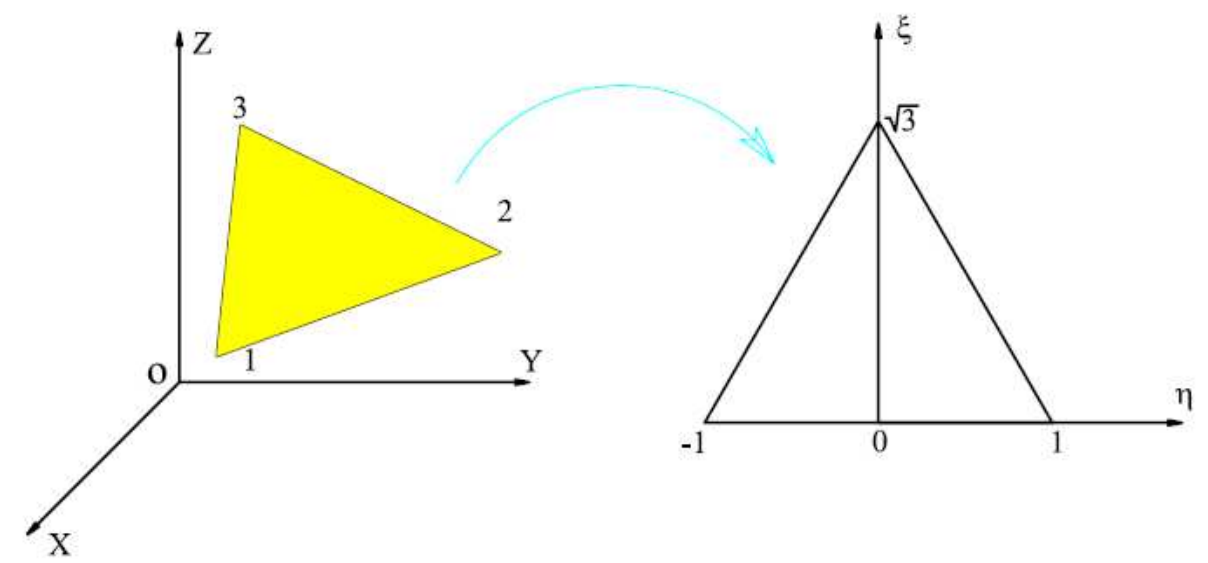

Fig. 20 Transformation of an element from global coordinate system to reference element

These functions could be rewritten in the vector form:

$$
\psi_{k}(\underline{\xi})=\lambda_{k}+\underline{\alpha}_{k} \underline{\xi} \quad(k=1,2,3)
$$

where, $\underline{\xi}=(\eta, \xi) ; \quad \underline{\alpha}_{k}=\left(\alpha_{k}, \beta_{k}\right) ; \quad \lambda_{1}=\lambda_{2}=0.5 ; \lambda_{3}=0 ; \quad \alpha_{1}=-0.5 ; \alpha_{2}=0.5 ; \alpha_{3}=0 ;$ 
$\beta_{1}=\beta_{2}=-\frac{1}{2 \sqrt{3}} ; \beta_{3}=\frac{1}{\sqrt{3}}$.

The transformation from a triangular element in the global coordinate system to a reference element is described as:

$$
\left(\begin{array}{l}
\eta \\
\xi
\end{array}\right)=J \cdot\left(\begin{array}{l}
x-x_{0} \\
y-y_{0}
\end{array}\right) \quad \text { or } \quad \underline{\xi}=J \cdot\left(\underline{x}-\underline{x}_{0}\right)
$$

with, $\boldsymbol{J}$ the matrix of transformation and $\left(x_{0}, \mathrm{y}_{0}\right)$ the origin of transformation. The shape functions thus are obtained in the global coordinate system:

$$
\psi_{k}(\underline{x})=\lambda_{k}+\underline{\alpha}_{k} J \cdot\left(\underline{x}-\underline{x}_{0}\right) \quad(k=1,2,3)
$$

Interpolation functions of pressure on the elements of type 1 and 2 can be deduced from equation (3). Their formulations in reference coordinate systems are given in equations (49) and (50), respectively. As a consequence, the extension of these formulations in global coordinates systems is carried out by using the following equation.

$$
\begin{aligned}
& \psi_{k}^{1}(\eta, \xi)=\lambda_{k}^{1}+\left(\begin{array}{l}
\alpha_{k}^{1} \\
\beta_{k}^{1}
\end{array}\right) \cdot\left(\begin{array}{c}
\eta \\
\xi \sqrt{\xi}
\end{array}\right) \quad(k=1,2,3) \\
& \psi_{k}^{2}(\eta, \xi)=\lambda_{k}^{2}+\left(\begin{array}{l}
\alpha_{k}^{2} \\
\beta_{k}^{2}
\end{array}\right) \cdot\left(\begin{array}{c}
\eta \\
\sqrt{(\sqrt{3}-\xi)^{3}}
\end{array}\right) \quad(k=1,2,3)
\end{aligned}
$$

with, $\lambda_{k}^{1}=\lambda_{k} ; \lambda_{1}^{2}=\lambda_{2}^{2}=0 ; \lambda_{3}^{2}=1 ; \alpha_{k}^{1}=-\alpha_{k}^{2}=\alpha_{k}$;

$\beta_{1}^{1}=\beta_{2}^{1}=-\beta_{1}^{2}=-\beta_{2}^{2}=-\frac{1}{2 \sqrt{3 \sqrt{3}}} ; \beta_{3}^{1}=-\beta_{3}^{2}=\frac{1}{\sqrt{3 \sqrt{3}}}$. 